This is the final peer-reviewed accepted manuscript of:

Alves, L., Hirata, S., Schuabb, M., \& Barletta, A. (2019). Identifying linear absolute instabilities from differential eigenvalue problems using sensitivity analysis. Journal of Fluid Mechanics, 870, 941-969

The final published version is available online at:

https://doi.org/10.1017/ifm.2019.275

Rights / License:

The terms and conditions for the reuse of this version of the manuscript are specified in the publishing policy. For all terms of use and more information see the publisher's website.

This item was downloaded from IRIS Università di Bologna (https://cris.unibo.it/)

When citing, please refer to the published version. 


\title{
Identifying Linear Absolute Instabilities from Differential Eigenvalue Problems using Sensitivity Analysis
}

\author{
L. S. de B. Alves ${ }^{1} \dagger$, S. C. Hirata ${ }^{2}$, M. Schuabb ${ }^{1}$ and A. Barletta ${ }^{3}$ \\ ${ }^{1}$ TEM, PGMec, Universidade Federal Fluminense, Brazil \\ ${ }^{2}$ Unité de Mécanique de Lille, UML EA 7512, F-59000, Lille, France \\ ${ }^{3}$ Dipartimento di Ingegneria Energetica, Alma Mater Studiorum, Università di Bologna, Italy
}

(Received xx; revised $\mathrm{xx}$; accepted $\mathrm{xx}$ )

Identifying the convective/absolute instability nature of a local base flow requires an analysis of its linear impulse response. One must find the appropriate branch point singularity in the dispersion relation with complex frequency/wavenumbers and prove causality. One way to do so is to show that the appropriate integration contour of this response, a steepest decent path through the relevant singularity, exists. Due to the inherent difficulties of such a proof, one often verifies instead if this singularity satisfies the collision criterion. In other words, one must show that the branches involved in the formation of this singularity come from distinct halves of the complex wavenumber plane. However, this graphical search is computationally intensive in a single plane and essentially prohibitive in two planes. A significant computational cost reduction can be achieved when root finding procedures are applied instead of graphical ones to search for singularities. They focus on locating these points, with causality being verified graphically a posteriori for a small parametric sample size. The use of root finding procedures require auxiliary equations, often derived by applying the zero group velocity conditions to the dispersion relation. This relation, in turn, is derived by applying matrix forming to the differential eigenvalue problem and taking the determinant of the resulting system of algebraic equations. Taking the derivative of the dispersion relation with respect to the wavenumbers generates the auxiliary equations. If the algebraic system is decoupled, this derivation is straightforward. However, its symbolic computational cost is often prohibitive when the algebraic system is coupled. This paper describes an alternative methodology based on sensitivity analysis and adjoints that allow the zero group velocity conditions to be applied directly to the differential eigenvalue problem. In doing so, the direct and auxiliary differential eigenvalue problems can be solved simultaneously using standard shooting methods to directly locate branch point singularities, dramatically reducing the costs associated with their graphical search while also eliminating the need to derive the auxiliary dispersion relations using symbolic computation. The search for arbitrary branch point singularities is then not only accelerated in single wavenumber planes but it also becomes viable in two wavenumber planes. Finally, the new method also allows group velocity calculations, greatly facilitating the verification of causality. Several test cases are presented to illustrate its capabilities. 


\section{Introduction}

When open dynamical systems are unstable, small amplitude disturbances superposed to a local base state are allowed to grow in space and/or time. In order to distinguish between these cases, one must identify the linear nature of the instability mechanism. This is achieved by analysing the asymptotic behaviour of wave packets generated by an impulse excitation of the linear and modal disturbance governing equation. Three possible scenarios emerge. If the disturbance envelope amplitude decays as it is advected downstream by the local base state, the system is convectively stable. Otherwise, it is unstable. In the latter case, two possible scenarios remain. If the disturbance envelope amplitude eventually decays in time when measured at a fixed location, the system is convectively unstable. Otherwise, the disturbance envelope amplitude will grow in time at any fixed location, which means the system is absolutely unstable. This understanding has an important impact on the development of control strategies for open dynamical systems. When these systems are convectively unstable, all disturbances eventually leave the domain of interest. In the absence of an external forcing that acts as a disturbance source, they eventually return to their original local base states. Hence, these systems are highly susceptible to control through low amplitude forcing. On the other hand, absolutely unstable systems allow any initially present disturbance to eventually grow in time, leading them away from their original local base state. In such cases, these systems become self-excited and more complex control strategies are required (Pier 2009).

Within the context of hydrodynamic instabilities, Betchov \& Criminale (1966) were among the first to find singularities in an eigenvalue problem when studying the linear stability of inviscid jets and wakes. Soon afterwards, Gaster (1968) recognised that these singularities appeared as saddle points in a complex wavenumber plane when analyzing the impulse response of the eigenvalue problem. Furthermore, he noticed that the wave packet generated by this pulse was physically meaningful when its group velocity was zero, which could indicate when spatially growing disturbances would start growing in time. These ideas were first applied to distinguish between convective and absolute instabilities in fluid dynamics by Thacker (1976) for the analysis of Gulf streams as well as Merkine (1977) for the study of baroclinic eddies. However, they did not develop these ideas and concepts, but instead drew from the theoretical foundations underlying these notions that were already extensively developed in the field of plasma instabilities. Popular among them were the collision criteria devised by Briggs (1964) and the convective/absolute instability description by Bers (1975). A detailed account of this development in plasma instabilities was reviewed by Bers (1983) whereas a survey on the use of these tools in the field of hydrodynamic instabilities was written by Huerre \& Monkewitz (1990).

The original demonstration of a transition to absolute instability required proof of causality, i.e. proof that the contours of the integration that defines the linear impulse response of a system can be deformed into a steepest descent integration path containing the branch point singularity. Due to the inherent difficulties in this proof, early attempts to find the parametric conditions where a local base state transitions from convectively to absolutely unstable relied on the collision criterion instead. This graphical proof was used to locate saddle points on the complex wavenumber plane that were in fact pinching points, i.e. that were formed by the collision between downstream and upstream propagating branches that satisfy the eigenvalue problem. The most common paths towards this graphical proof of causality are illustrated in Fig. 1, where white and light blue boxes represent theoretical and numerical steps, respectively. Paths $D_{1}$ and $D_{2}$ in this figure respectively indicate the use of matrix forming and shooting methods to solve differential eigenvalue problems in order to evaluate the collision criterion. 


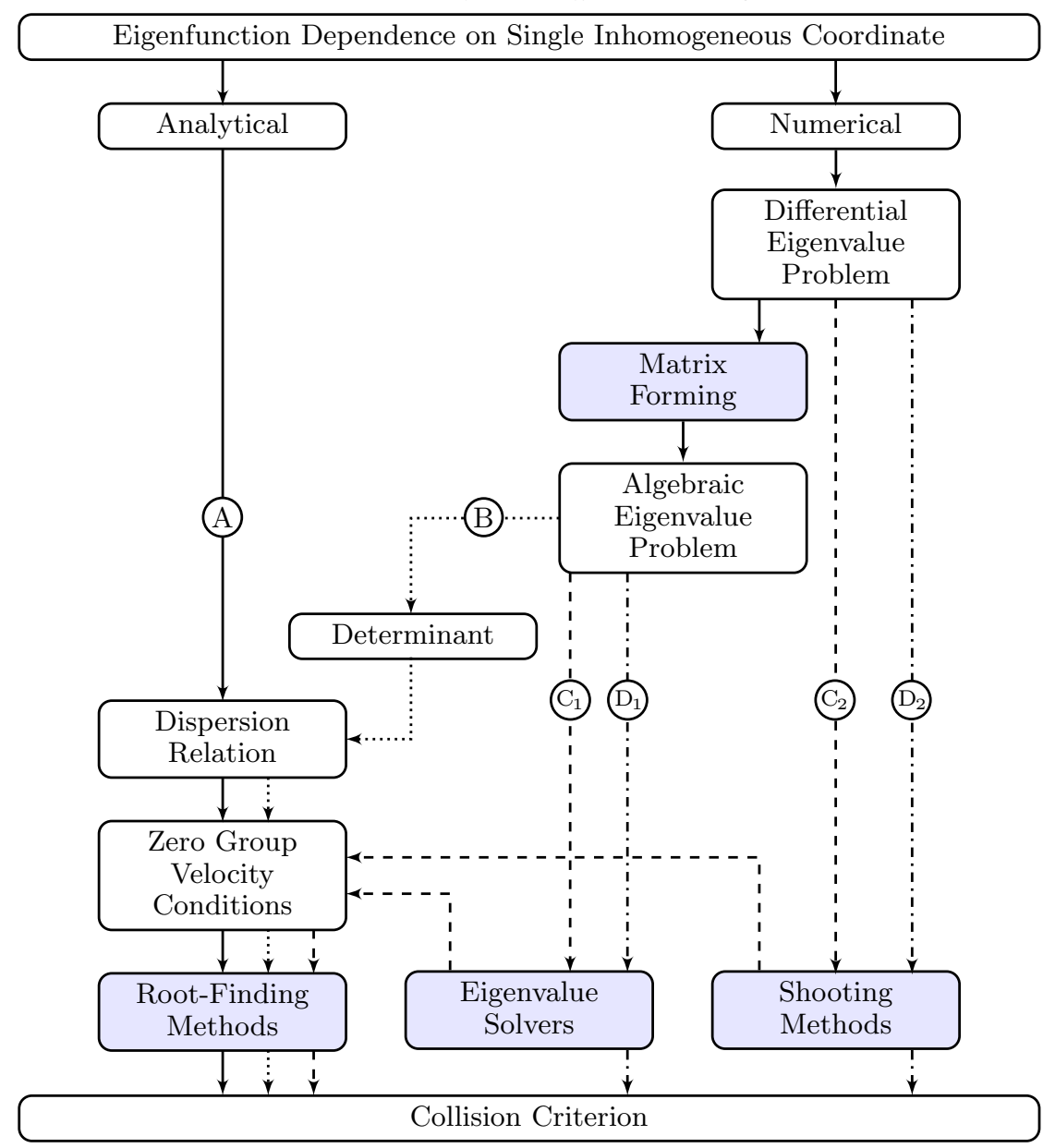

FiguRE 1. Local and Linear Absolute Stability Theory and Numerics Flowchart.

The latter has been used in linear stability analyses of planar mixing layers (Huerre \& Monkewitz 1985), flows around bluff bodies (Monkewitz \& Nguyen 1987), hot jets (Monkewitz \& Sohn 1988), boundary layers on rotating disks (Lingwood 1995, 1997a), and several other problems. A common feature among these studies is their focus on twodimensional instabilities. This terminology is used here to emphasize that wave packets are allowed to propagate in a single homogenous direction. Since only one direction is inhomogeneous in a local analysis, any variation in the remaining homogeneous direction is defined by its respective wavenumber. The former three references prescribe it to zero whereas the latter two references prescribe it to a positive number. For this reason, the latter two are named pseudo two-dimensional absolute instability analyses from this point on. Nevertheless, any search for branch points always considers only a single wavenumber plane when dealing with two or pseudo-two dimensional instabilities. The extension of this transition framework towards three-dimensional instabilities was derived by Brevdo (1991). In this case, wave packets are allowed to propagate in both homogeneous directions and the graphical search for branch points must consider both wavenumber planes simultaneously. However, he noted the greater difficulty in proving the existence of a steepest descent surface, as compared to its line counterpart in twodimensions, recommending the application of the collision criterion instead. Nevertheless, 
even this criterion is numerically expensive in two-dimensions and prohibitive in threedimensions. It is much cheaper to only search for branch point singularities using the zero group velocity conditions, which can be done using a asymptotic expansions that are known since the work of Benjamin (1961) and Criminale \& Kovasznay (1962), without performing a collision check to verify causality. This led to the development of techniques that track the movement of singularities as one parameter is varied, where the most popular ones are known as continuation methods. Well known examples of such methods were developed by Deissler (1987), Brevdo (1995) and Brevdo et al. (1999) for two-dimensional instabilities and extended by Oertel Jr. \& Delfs (1995) and Koch (2002) to three-dimensional instabilities. They numerically calculate the group velocities, often based on the frequency dependence on the wavenumbers obtained from a temporal stability analysis, and locate the branch points with root-finding methods. It is important to note that continuation methods perform their temporal stability calculations using either algebraic (with eigenvalue solvers) or differential (with shooting methods) eigenvalue problems, illustrated by paths $C_{1}$ and $C_{2}$ in Fig. 1, respectively. Causality is verified a posteriori for a small sample of branch points in order to reduce computational cost. Nevertheless, this sample is usually chosen large enough to generate sufficient confidence that the entire set satisfies causality. A review of continuation methods was presented by Suslov (2006), who also proposed a more efficient variation, but only two-dimensional calculations were presented. Despite their widespread use, a word of caution about collision criterion/steepest descent methods and continuation methods is required. Lingwood (1997b) has shown that it might not be an easy task to prove that the inversion contour can be deformed into the steepest descent path for any given branch point. Furthermore, Brevdo et al. (1999) have shown that continuation methods must be used with care because they cannot capture branch points that are not connected by continuity. These difficulties in the search for three-dimensional singularities often force researchers to search instead for two-dimensional (zero either streamwise or spanwise waven umber) ones, as done by Hirata et al. (2015), or pseudo two-dimensional (nonzero but real spanwise wavenumber) ones, as done by Lingwood (1997c). However, this compromise can lead to misleading results. Brevdo \& Ruderman (2009) found in their study of mixed convection in a porous medium that two-dimensional longitudinal (zero streamwise wavenumber) modes became absolutely unstable under the same conditions that Brevdo (2009) found three-dimensional longitudinal modes to always be convectively unstable. Furthermore, Alves \& Barletta (2015) found in a similar problem, but for dilatant fluids modeled by a power-law, that the onset of absolute instability switched in a discontinuous manner between transversal (zero spanwise wavenumber) and longitudinal modes as the Péclet number was increased in a three-dimensional instability analysis. On the other hand, they found that this switch was smooth, going through all possible oblique modes in between, in a pseudo two-dimensional instability analysis. All these cautionary examples show the importance of distinguishing three, pseudo two and two-dimensional branch point singularities.

As mentioned by Koch (2002), the numerical difficulties associated with continuation methods are an issue because they are the best known methods available for the search of three-dimensional singularities when dealing with differential eigenvalue problems. This difficulty in locating three-dimensional absolutely unstable branch points even affects the WKBJ reconstruction of global modes, according to Martinand et al. (2004). Whenever a dispersion relation can be found, auxiliary dispersion relations can be derived using zero group velocity conditions and the search for branch points reduces to solving the resulting system of algebraic equations with root finding methods. Many examples can be found in the literature, such as the studies by Chomaz et al. (1999), Delache et al. 
(2007), Hirata \& Ouarzazi (2010) and Alves \& Barletta (2015). These examples share a common feature, their dispersion relations are simple analytical expressions due to the analytical form of their eigenfunction dependence on their single inhomogeneous coordinate. This is the path A illustrated in Fig. 1. The use of such auxiliary dispersion relations is so effective that some researchers try and avoid the use of steepest descent, collision or continuation methods to search for branch points from differential eigenvalue problems. They use instead a matrix forming approach to transform the differential eigenvalue problem into an algebraic eigenvalue problem. Requiring its determinant to be zero for a nontrivial solution to exist also leads to a dispersion relation, although they are significantly more complex analytical expressions. In doing so, the zero group velocity conditions can once again be used to generate auxiliary dispersion relations and the search for branch points can be performed with root finding methods. Such a matrix forming approach was employed by Carrière \& Monkewitz (1999) using Galerkin/collocation methods and recently in a still ongoing study by Brandao et al. (2014) and Brandao et al. (2015) using a generalized Fourier transform. This is the path B illustrated in Fig. 1. However, as noted by Brevdo (2009), solving this system of equations can be a rather involved computational task. In fact, Hirata et al. (2015) attempted to solve their problem through path B using a Galerkin method based matrix forming, but the very high symbolic computational cost associated with deriving the auxiliary dispersion relations using the zero group velocity conditions did not allow numerically converged results to be reached. These examples reveal that a reliable method to extract three-dimensional branch points from differential eigenvalue problems is yet to be found.

In the present paper, a novel procedure is put forward that enables the search for two and three-dimensional branch points in differential eigenvalue problems without having to resort to determinants (path B), continuation methods (paths C) or collision checks (paths D). It applies the ideas associated with adjoint sensitivity analysis (Luchini \& Bottaro 2014) to boundary value problems so the zero group velocity conditions can be used to generate auxiliary differential eigenvalue problems, analogous to the auxiliary dispersion relations from path $\mathrm{A}$. This coupled system of equations now contains two and three differential eigenvalue problems for two and three-dimensional branch point searches, respectively, which can be solved using the same shooting methods used to solve the direct differential eigenvalue problem (in paths $C_{2}$ and $D_{2}$ ). When coupled with adjoint analysis (Luchini \& Bottaro 2014), this procedure can also calculate the group velocity of the modes within both spatial branches involved in the collision that forms the branch point. In doing so, it can determine the propagating directions of these two and three-dimensional branches involved in the collision. Well known two, pseudo two and three-dimensional instabilities are solved to demonstrate the capabilities of this novel procedure. Fluid properties are considered constant in these test cases, but allowing them to vary does not modify the proposed methodology in any significant way.

\section{Mathematical Formulation}

\subsection{Differential Eigenvalue Problems}

Consider an open dynamical system in the form of

$$
\frac{\partial \mathbf{q}}{\partial t}=\mathbf{f}(\mathbf{q})
$$


where $\mathbf{f}\left(\mathbf{q}_{b}\right)=0$ yields its nonlinear steady-state and its state variable vector $\mathbf{q}(\mathbf{x}, t)$ can be decomposed into the usual local, one-dimensional and modal form

$$
\mathbf{q}(\mathbf{x}, t)=\mathbf{q}_{b}(z)+\epsilon \mathbf{q}_{n}(z) e^{i(\alpha x+\beta y-\omega t)},
$$

where $\epsilon$ is a disturbance amplitude parameter, $i$ is the imaginary unit, $x$ and $y$ are the streamwise and spanwise coordinates, respectively, $z$ is the only inhomogeneous coordinate and $t$ is time. Furthermore, the local base state vector is $\mathbf{q}_{b}(z)$ and the disturbance normal mode is defined by its eigenfunction vector $\mathbf{q}_{n}(z)$ and its eigenvalues, given by the frequency $\omega$ as well as streamwise and spanwise wavenumbers $\alpha$ and $\beta$, respectively. When substituting decomposition (2.2) into the dynamical system governing equation (2.1) and collecting the linear terms of $O(\epsilon)$, an ordinary differential system of equations with respect to $z$ is obtained for $\mathbf{q}_{n}(z)$. It is written here as

$$
\mathcal{L} \mathbf{q}_{n}=0,
$$

where $\mathcal{L}=\mathcal{L}(\alpha, \beta, \omega ; \mathcal{R})$ is a linear and homogeneous differential operator that depends on all eigenvalues and $\mathcal{R}$, which can represent either one or more control parameters. Examples include the Reynolds, Prandtl, Péclet and Rayleigh numbers. Equation (2.3) could also be a partial differential equation, if one assumes $\mathbf{q}(\mathbf{x}, t)=$ $\mathbf{q}_{b}(y, z)+\epsilon \mathbf{q}_{n}(y, z) e^{i(\alpha x-\omega t)}$ instead of Eq. (2.2). Under such constraints, a local but two-dimensional modal analysis would be performed, although it can also be known as a spatial Bi-Global analysis in the literature (Theofilis 2003, 2011). An example of such a local two-dimensional analysis is the case of an eccentric Taylor-CouettePoiseuille flow recently studied by Leclercq et al. (2014). In any case, Eq. (2.3) is still a differential eigenvalue problem. The different information about two, pseudo two and three-dimensional branch point singularities, as well as group velocities, one can extract from this equation using shooting methods is the focus of the present paper.

\subsection{Dispersion Relations}

Matrix forming Eq. (2.3) can be used instead to generate dispersion relations. However, this approach is often unfeasible and, hence, should be avoided when searching for linear absolute instabilities. In order to better illustrate why, path B from Fig 1 is discussed now. Applying an arbitrary spatial discretization to Eq. (2.3) yields

$$
L q_{n}=0,
$$

where $L \in \mathbb{C}^{(N \times N)}$ and $q_{n} \in \mathbb{C}^{(N)}$ are the discrete and complex matrix and eigenvector versions of differential operator $\mathcal{L}$ and eigenfunction $\mathbf{q}_{n}$, respectively, with $N$ defining the arbitrary size of this system. The first dispersion relation comes from the necessary condition for the existence of non-trivial solutions for $q_{n}$ in Eq. (2.4), i.e.

$$
\operatorname{det}(L)=0,
$$

where $\operatorname{det}(L)$ is the determinant of $L$. The auxiliary dispersion relations needed to search for branch point singularities then come from

$$
\begin{array}{llll}
\frac{\partial \operatorname{det}(L)}{\partial \alpha}=0 & \text { with } & \frac{\partial \omega}{\partial \alpha}=0 \quad \text { and } \\
\frac{\partial \operatorname{det}(L)}{\partial \beta}=0 & \text { with } & \frac{\partial \omega}{\partial \beta}=0 \quad,
\end{array}
$$

for two, pseudo two and three-dimensional instabilities. Whenever Eq. (2.3) is normal, i.e. $\mathbf{q}_{n}(z)$ has a modal dependence on $z, L$ is a diagonal matrix. In this case, $\operatorname{det}(L)$ is the 
product of its diagonal elements, i.e. the algebraic eigenvalue problem defined in Eq. (2.4) is a decoupled system of algebraic equations. Hence, the symbolic computational cost of generating Eqs. (2.5) to (2.7) is essentially negligible. On the other hand, if Eq. (2.3) is non-normal, $L$ is no longer diagonal and the algebraic eigenvalue problem now represents a coupled system of equations. Hence, the symbolic computational cost of generating $\operatorname{det}(L)$ and its derivatives with respect to the wavenumbers is much higher. In fact, this cost can be prohibitively high even for moderate degrees of coupling and model complexity, as mentioned in section 1 . Numerically evaluating this determinant and its derivatives instead usually speeds up computations, but in general this is not enough to allow path $\mathrm{B}$ to be competitive with paths $\mathrm{C}$ and $\mathrm{D}$ in Fig. 1.

\subsection{Sensitivity Analysis}

In order to avoid the potentially prohibitive symbolic/numeric computational cost associated with deriving the dispersion relations required for the search of branch points using Eq. (2.5), one must search for them using differential eigenvalue problems in the form of Eq. (2.3) instead. In the present paper, a shooting method is employed to solve this equation. Doing so yields two complex solutions: the eigenfunction vector $\mathbf{q}_{n}(z)$ and one eigenvalue, for prescribed values of the remaining eigenvalues. All control parameters are prescribed as well. When performing a temporal stability analysis, $\omega$ is the complex eigenvalue being solved for prescribed real values of the remaining eigenvalues $\alpha$ and $\beta$. On the other hand, when performing a spatial stability analysis, $\alpha$ is the complex eigenvalue being solved for prescribed real values of the remaining eigenvalues $\omega$ and $\beta$.

Since $\omega, \alpha$ and $\beta$ are coupled at these branch points, auxiliary differential eigenvalue problems must be solved simultaneously with Eq. (2.3) for these eigenvalues. Taking the derivative of this equation with respect to $\alpha$ and $\beta$, respectively, leads to

$$
\begin{gathered}
\mathcal{L} \mathbf{q}_{\alpha}=\mathcal{S}_{\alpha} \quad \text { with } \quad \mathcal{S}_{\alpha}=-\mathcal{L}_{\alpha} \mathbf{q}_{n} \quad \text { and } \\
\mathcal{L} \mathbf{q}_{\beta}=\mathcal{S}_{\beta} \quad \text { with } \quad \mathcal{S}_{\beta}=-\mathcal{L}_{\beta} \mathbf{q}_{n},
\end{gathered}
$$

where $\mathbf{q}_{\alpha}=\partial \mathbf{q}_{n} / \partial \alpha, \mathbf{q}_{\beta}=\partial \mathbf{q}_{n} / \partial \beta, \mathcal{L}_{\alpha}=\partial \mathcal{L} / \partial \alpha$ and $\mathcal{L}_{\beta}=\partial \mathcal{L} / \partial \beta$. Furthermore, $\mathcal{L}_{\alpha}=$ $\mathcal{L}_{\alpha}(\alpha, \beta, \omega, \partial \omega / \partial \alpha ; \mathcal{R}, \partial \mathcal{R} / \partial \alpha)$ and $\mathcal{L}_{\beta}=\mathcal{L}_{\beta}(\alpha, \beta, \omega, \partial \omega / \partial \beta ; \mathcal{R}, \partial \mathcal{R} / \partial \beta)$ are also linear and homogeneous operators, where $\mathbf{q}_{n}(z), \omega$ and $\mathcal{R}$ were allowed to depend on $\alpha$ and $\beta$. The latter dependence is included here to generalize this procedure to calculate critical points for the onset of convective instabilities (Alves et al. 2016), even though this paper is focussed on absolute instabilities. Equations (2.8) and (2.9) are solved within the same shooting methodology used to solve for Eq. (2.3). Since they also yield two complex solutions each, one eigenfunction and one eigenvalue, these three equations can be used to find three eigenfunctions and three eigenvalues for three-dimensional instabilities. Two eigenvalues and two eigenfunctions can similarly be found for two and pseudo two-dimensional instabilities, where either Eq. (2.8) or (2.9) is dropped.

At this point, it is important to justify our focus on differential equations and shooting methods instead of matrix forming and generalized eigenvalue solvers. Both approaches are widely used in the literature. The former solves Eq. (2.3) whereas the latter solves Eq. (2.4), usually in the form of $A q_{n}=\omega B q_{n}$ for a temporal stability analysis. A nonlinear eigenvalue problem appears, however, if a spatial stability analysis is desired instead. For instance, a typical example of the form Eq. (2.4) takes in such an analysis is $A q_{n}=\alpha B_{1} q_{n}+\alpha^{2} B_{2} q_{n}$. This requires linearization, which is achieved with the companion matrix method (Bridges \& Morris 1984). Matrixes $A$ and $B$ come from $L$. In order to directly solve for branch points using this approach, however, one needs to apply matrix forming to Eqs. (2.8) and (2.9). Doing so to the former leads to Eq. (2.23), 
discussed later on in a different context. This yields a system of three coupled generalized eigenvalue problems with three different but coupled eigenvalues. At the present time, it is not clear how such a problem can be solved in an efficient manner.

Although Eqs. (2.8) and (2.9) were obtained from Eq. (2.3) in the same way Eqs. (2.6) and (2.7) were obtained from Eq. (2.5), their derivation is closely related to sensitivity analysis (Luchini \& Bottaro 2014). In such an analysis, one introduces small variations in a generalized eigenvalue problem, usually in the form of Eq. (2.4), and uses the adjoint eigenvector $q_{n}^{+}$of the direct eigenvector $q_{n}$ to identify gradients between different problem variables and/or parameters. Equations (2.8) and (2.9) can then be understood as the differential version of this analysis, based on Eq. (2.3) instead, where gradients with respect to the wavenumbers are sought. Although sensitivity studies of global modes are based on algebraic equations, their differential counterparts have been used for similar studies of local (Bottaro et al. 2003) and global modes (Juniper \& Pier 2015). The present method, however, is distinct in two ways. The first one is that the adjoint eigenfunction $\mathbf{q}_{n}^{+}(z)$ of the direct eigenfunction $\mathbf{q}_{n}(z)$ does not have to be obtained in order to solve for Eqs. (2.8) and (2.9), but wavenumber sensitivity eigenfunctions $\mathbf{q}_{\alpha}(z)$ and $\mathbf{q}_{\beta}(z)$ have to be obtained instead. The second way is that a normalisation, introduced in a traditional sensitivity analysis based on $q_{n}^{+}(z)$, is also required in Eqs. (2.8) and (2.9). Since they are also eigenvalue problems, $\mathbf{q}_{\alpha}(\mathrm{z})$ and $\mathbf{q}_{\beta}(z)$ are undetermined with respect to one integration constant each, which means normalisations are required to remove them.

Adjoints may not be required to solve Eqs. (2.8) and (2.9), but they are necessary to guarantee that a solution to these equations exists. Using $\langle u, v\rangle$ as the notation for an yet to be defined inner product between two arbitrary complex functions $u(z)$ and $v(z)$, an adjoint differential eigenvalue problem operator $\mathcal{L}^{*}$ can be defined using either

$$
\begin{aligned}
& \left\langle\mathcal{L} \mathbf{q}_{\alpha}, \mathbf{q}_{n}^{+}\right\rangle=\left\langle\mathbf{q}_{\alpha}, \mathcal{L}^{*} \mathbf{q}_{n}^{+}\right\rangle \text {or } \\
& \left\langle\mathcal{L} \mathbf{q}_{\beta}, \mathbf{q}_{n}^{+}\right\rangle=\left\langle\mathbf{q}_{\beta}, \mathcal{L}^{*} \mathbf{q}_{n}^{+}\right\rangle,
\end{aligned}
$$

where $*$ means conjugate transpose, to produce $\mathbf{q}_{n}^{+}(z)$, an adjoint of $\mathbf{q}_{n}(z)$, from

$$
\mathcal{L}^{*} \mathbf{q}_{n}^{+}=0,
$$

which is the adjoint version of Eq. (2.3). Using the linear and inhomogeneous Eqs. (2.8) and (2.9), as well as Eq. (2.12), it is possible to simplify Eqs. (2.10) and (2.11) to

$$
\begin{aligned}
& \left\langle\mathcal{S}_{\alpha}, \mathbf{q}_{n}^{+}\right\rangle=0 \quad \text { and } \\
& \left\langle\mathcal{S}_{\beta}, \mathbf{q}_{n}^{+}\right\rangle=0 \quad,
\end{aligned}
$$

known as solvability conditions. They derive from the Fredholm alternative theorem, which states that the inhomogeneous term of a linear equation, e.g. $\mathcal{S}_{\alpha}$ or $\mathcal{S}_{\beta}$, must be orthogonal to the adjoint solution of the corresponding homogeneous equation, e.g. $\mathbf{q}_{n}^{+}$, for the existence of nontrivial solutions to the inhomogeneous equation, e.g. $\mathbf{q}_{\alpha}$ or $\mathbf{q}_{\beta}$.

Equations (2.13) and (2.14) can now be used to calculate the group velocities $\partial \omega / \partial \alpha$ and $\partial \omega / \partial \beta$, which naturally appear within $\mathcal{L}_{\alpha}$ and $\mathcal{L}_{\beta}$, respectively. Since these operators can always be re-written as $\mathcal{L}_{\alpha}=\mathcal{L}_{\alpha}^{(0)}-\mathcal{L}_{\alpha}^{(1)} \partial \omega / \partial \alpha$ and $\mathcal{L}_{\beta}=\mathcal{L}_{\beta}^{(0)}-\mathcal{L}_{\beta}^{(1)} \partial \omega / \partial \beta$, Eqs. (2.13) and (2.14) can be correspondingly re-written to yield

$$
\begin{aligned}
\frac{\partial \omega}{\partial \alpha} & =\frac{\left\langle\mathcal{L}_{\alpha}^{(0)} \mathbf{q}_{n}, \mathbf{q}_{n}^{+}\right\rangle}{\left\langle\mathcal{L}_{\alpha}^{(1)} \mathbf{q}_{n}, \mathbf{q}_{n}^{+}\right\rangle} \quad \text { and } \\
\frac{\partial \omega}{\partial \beta} & =\frac{\left\langle\mathcal{L}_{\beta}^{(0)} \mathbf{q}_{n}, \mathbf{q}_{n}^{+}\right\rangle}{\left\langle\mathcal{L}_{\beta}^{(1)} \mathbf{q}_{n}, \mathbf{q}_{n}^{+}\right\rangle},
\end{aligned}
$$


which is the differential eigenvalue problem equivalent of the formula derived by Lesshafft \& Marquet (2010) from Eq. (2.4) in their hot jet base flow optimisation study.

In certain cases, however, it is possible to avoid the adjoint problem altogether, greatly simplifying group velocity calculations. Inner products satisfy conjugate symmetry, i.e. $\langle u, v\rangle=\overline{\langle v, u\rangle}$, where overbar means complex conjugate. This allows one to also define

$$
\left\langle\mathcal{L} \mathbf{q}_{n}, \mathbf{q}_{n}^{+}\right\rangle=\overline{\left\langle\mathcal{L}^{*} \mathbf{q}_{n}^{+}, \mathbf{q}_{n}\right\rangle},
$$

which is equal to zero as well. Using the fact that any two complex functions satisfy the multiplication property $\overline{u v}=\bar{u} \bar{v}$, it is possible to show that $\mathbf{q}_{n}^{+}=\overline{\mathbf{q}}_{n}$ satisfies Eq. (2.17) when $\mathcal{L}$ is a symmetric operator, i.e. $\mathcal{L}=\mathcal{L}^{T}=\overline{\mathcal{L}^{*}}$. The superscript $T$ means transpose. Such a restriction might be alleviated if it is possible to define a linear operator $\mathcal{P}$ in such a way that $\mathcal{P} \mathcal{L}=(\mathcal{P} \mathcal{L})^{T}=\overline{(\mathcal{P} \mathcal{L})^{*}}$. Applying it to Eq. $(2.3)$, and all subsequent derivations, necessarily modifies the solvability conditions in Eqs. (2.13) and (2.14) to

$$
\begin{aligned}
& \left\langle\mathcal{P} \mathcal{S}_{\alpha}, \overline{\mathbf{q}}_{n}\right\rangle=0 \quad \text { and } \\
& \left\langle\mathcal{P} \mathcal{S}_{\beta}, \overline{\mathbf{q}}_{n}\right\rangle=0 \quad,
\end{aligned}
$$

removing the adjoint eigenfunction from them. In other words, the inhomogeneous term of a symmetric linear equation, e.g. $\mathcal{P} \mathcal{S}_{\alpha}$ or $\mathcal{P} \mathcal{S}_{\beta}$, must be orthogonal to the conjugate solution of the corresponding homogeneous equation, e.g. $\overline{\mathbf{q}}_{n}$, for the existence of nontrivial solutions to the inhomogeneous equation, e.g. $\mathbf{q}_{\alpha}$ or $\mathbf{q}_{\beta}$. This is always the case for second $(s=0)$ and fourth $(s \neq 0)$ order operators that can be written as

$$
\mathcal{P} \mathcal{L}=q-D(p D)+D^{2}\left(s D^{2}\right),
$$

with suitable boundary conditions, where $D=d / d z$ and $q(z), p(z)$ and $s(z)$ are problem dependent arbitrary complex functions. Such an orthogonality condition has been used by Alves et al. (2008) and Kelly \& Alves (2008) in their study of convectively unstable jets in cross flow. In this particular case, Eqs. (2.15) and (2.16) can be re-written as

$$
\begin{aligned}
\frac{\partial \omega}{\partial \alpha} & =\frac{\left\langle\mathcal{P} \mathcal{L}_{\alpha}^{(0)} \mathbf{q}_{n}, \overline{\mathbf{q}}_{n}\right\rangle}{\left\langle\mathcal{P} \mathcal{L}_{\alpha}^{(1)} \mathbf{q}_{n}, \overline{\mathbf{q}}_{n}\right\rangle} \quad \text { and } \\
\frac{\partial \omega}{\partial \beta} & =\frac{\left\langle\mathcal{P} \mathcal{L}_{\beta}^{(0)} \mathbf{q}_{n}, \overline{\mathbf{q}}_{n}\right\rangle}{\left\langle\mathcal{P} \mathcal{L}_{\beta}^{(1)} \mathbf{q}_{n}, \overline{\mathbf{q}}_{n}\right\rangle},
\end{aligned}
$$

which will be used in the first two test cases analyzed in section 3 to provide practical examples where symmetries can be explored to simplify group velocity calculations.

\subsection{Methodology Equivalency}

It is possible to show that solving auxiliary differential eigenvalue problems (2.8) and (2.9) is equivalent to solving auxiliary dispersion relations (2.6) and (2.7). Considering first the two-dimensional instability case, applying to Eq. (2.8) the same arbitrary discretization scheme used on Eq. (2.3) to matrix form Eq. (2.4) yields

$$
L q_{\alpha}+A q_{n}=0,
$$

where $A \in \mathbb{C}^{(N \times N)}$ and $q_{\alpha} \in \mathbb{C}^{(N)}$ are the discrete and complex matrix and eigenvector versions of operator $\mathcal{L}_{\alpha}$ and eigenfunction $\mathbf{q}_{\alpha}$, respectively. Multiplying Eq. (2.23) by $\operatorname{adj}(L)$ and using the identity $\operatorname{adj}(L) L=\operatorname{det}(L) I$ allows one to re-write it as

$$
\operatorname{det}(L) I q_{\alpha}+\operatorname{adj}(L) A q_{n}=0,
$$


where $I$ is the identity matrix and $\operatorname{adj}(L)$ is the adjugate matrix of $L$, which is defined as the transpose of the cofactor matrix of $L$ (Strang 1988). Equation (2.5) necessarily indicates that Eq. (2.24) can provide nontrivial solutions for $q_{n}$, due to the fact that $\operatorname{det}(\operatorname{adj}(L) A)=\operatorname{det}(\operatorname{adj}(L)) \operatorname{det}(A)=\operatorname{det}(L)^{N-1} \operatorname{det}(A)=0$. This result also shows that such a determinant cannot be used to generate the required auxiliary dispersion relations. One can, however, take the trace instead, obtaining

$$
\operatorname{tr}(\operatorname{adj}(L) A)=0,
$$

as a necessary condition for the existence of non-trivial solutions for $q_{n}$ in Eq. (2.24), subject to Eq. (2.5). Finally, Eq. (2.25) is equivalent to

$$
\frac{\partial \operatorname{det}(L)}{\partial \alpha}=0
$$

according to another identity known as Jacobi's formula. Hence, solving Eqs. (2.3) and (2.8) is equivalent to solving Eqs. (2.5) and (2.6). Extending this demonstration to the three-dimensional instability case is straightforward, since it leads to

$$
\operatorname{tr}(\operatorname{adj}(L) B)=\frac{\partial \operatorname{det}(L)}{\partial \beta}=0
$$

where $B \in \mathbb{C}^{N, N}$ is the discrete and complex matrix version of operator $\mathcal{L}_{\beta}$. This proof also indirectly shows that auxiliary differential eigenvalue problems (2.8) and (2.9) naturally reduce to the simple auxiliary dispersion relations obtained though path $\mathrm{A}$ in Fig 1 when the eigenfunction dependence on the inhomogeneous coordinate has a simple analytical form, since $L$ becomes a very sparsely coupled matrix and, hence, $\operatorname{det}(L)=0$ yields a simple algebraic equation as a dispersion relation.

\section{Results and Discussion}

This method just is now applied to problems with two, pseudo two and threedimensional instabilities found in test cases from the literature in the following subsections. Each problem will be written using a nomenclature similar to that of section 2. This is done in order to create some coherence between different verification tests. The shooting method employed here to generate the results used in the following figures was coded within the software Mathematica (Wolfram 2003). This code uses the built-in function NDSolve to numerically simulate the ordinary differential equations in the differential eigenvalue problems as initial value problems instead of boundary value ones. In its default method option Automatic, it uses variable marching steps and switches between different schemes, with different numerical stability and accuracy orders, in order to guarantee either absolute or relative errors requested through the control parameter WorkingPrecision. The eigenvalues and additional initial condition constants, created when transforming the boundary value problem into an initial value one, are obtained in order to satisfy the boundary conditions ignored in this transformation. This search is performed with the built-in function FindRoot, using Newton's method with a numerical Jacobian obtained by finite differences. The iteration stopping criterion is controlled by another WorkingPrecision parameter. Grid convergence is performed by increasing the value assigned to WorkingPrecision until the desired number of converged digits is achieved. More specific details about the general shooting method used here can be found in the papers by Alves et al. (2008), Kelly \& Alves (2008) and Hirata et al. (2015). 


\subsection{Two-Dimensional Branch Point Singularities}

The first test case reproduces the two-dimensional $(\beta=0)$ branch point calculations reported by Huerre \& Monkewitz (1985) for the planar mixing layer. This is the shear flow created when two parallel isothermal streams with different velocities meet after the end of the thin splitter plate separating them. Such a process generates an inflectional base state that is subject to an inviscid instability. It is convectively unstable when both streams flow in the same direction, but it becomes absolutely unstable when a sufficient amount of reverse flow is imposed. The local base state is defined on an infinite domain and described by the family of profiles given by

$$
U_{b}(z)=1+R \tanh \left[\frac{z}{2}\right],
$$

where $R$ is defined as the ratio between the difference and the sum of the velocities of both streams and $U_{b}(z)$ is the base flow streamwise velocity component. The direct differential eigenvalue problem, written in the form of Eq. (2.3) for the linear and modal pressure disturbance eigenfunction $P_{n}(z)$, is given by

$$
\mathcal{L} P_{n}=0 \quad \text { with } \quad \mathcal{L}=D^{2}+\frac{2 \alpha\left(D U_{b}\right)}{\omega-\alpha U_{b}} D-\alpha^{2}
$$

which is subject to the asymptotic boundary conditions

$$
P_{n}(z \rightarrow-\infty)=c_{1} e^{\alpha z} \text { and } P_{n}(z \rightarrow+\infty)=c_{2} e^{-\alpha z},
$$

where Eq. (3.2) is marched from the left (right) side of the domain to the centre of the mixing layer using initial conditions obtained from the left (right) boundary condition in Eq. (3.3), where infinity is a high enough value of $z$ to yield a negligible base state first derivative. This yields normalised solutions $\tilde{P}_{l}(z)=P_{n}(z) / c_{1}$ and $\tilde{P}_{r}(z)=P_{n}(z) / c_{2}$, respectively. The Newton-type root finding method can then solve the Wronskian

$$
f_{n}(\alpha, \omega)=\tilde{P}_{l}(0)\left(D \tilde{P}_{r}(0)\right)-\tilde{P}_{r}(0)\left(D \tilde{P}_{l}(0)\right)=0,
$$

avoiding the need to find constants $c_{1}$ and $c_{2}$.

Taking the derivative of Eq. (3.2) with respect to $\alpha$ while noting that $\partial U_{b} / \partial \alpha=0$ since $\partial R / \partial \alpha=0$, leads to re-writing the auxiliary problem in Eq. (2.8) as

$$
\mathcal{L} P_{\alpha}=\mathcal{S}_{\alpha}=-\mathcal{L}_{\alpha} P_{n} \quad \text { with } \quad \mathcal{L}_{\alpha}=\left(\omega-\alpha \frac{\partial \omega}{\partial \alpha}\right) \frac{2\left(D U_{b}\right)}{\left(\omega-\alpha U_{b}\right)^{2}} D-2 \alpha,
$$

where $P_{\alpha}(z)=\partial P_{n} / \partial \alpha$ is defined as an additional eigenfunction. This equation is subject to asymptotic boundary conditions

$$
\begin{aligned}
& P_{\alpha}(z \rightarrow-\infty)=\left(c_{3}+c_{1}\left(z-\frac{1}{2 \alpha}\right)\right) e^{+\alpha z} \text { and } \\
& P_{\alpha}(z \rightarrow+\infty)=\left(c_{4}-c_{2}\left(z+\frac{1}{2 \alpha}\right)\right) e^{-\alpha z}
\end{aligned}
$$

which can be further simplified by choosing $c_{3}=c_{1} /(2 \alpha)$ and $c_{4}=c_{2} /(2 \alpha)$, yielding the same boundary conditions one would obtain by taking the derivative of Eq. (3.3) with respect to $\alpha$ while neglecting the dependence of $c_{1}$ and $c_{2}$ on $\alpha$. This is possible because Eq. (3.5) is solved with the same procedure used to solve Eq. (3.2), where the constants disappear from the procedure by solving for the normalised solutions $\bar{P}_{l}(z)=P_{\alpha}(z) / c_{1}$ and $\bar{P}_{r}(z)=P_{\alpha}(z) / c_{2}$ instead. The new Wronskian

$$
f_{\alpha}(\alpha, \omega)=\bar{P}_{l}(0)\left(D \bar{P}_{r}(0)\right)-\bar{P}_{r}(0)\left(D \bar{P}_{l}(0)\right)=0 \quad,
$$



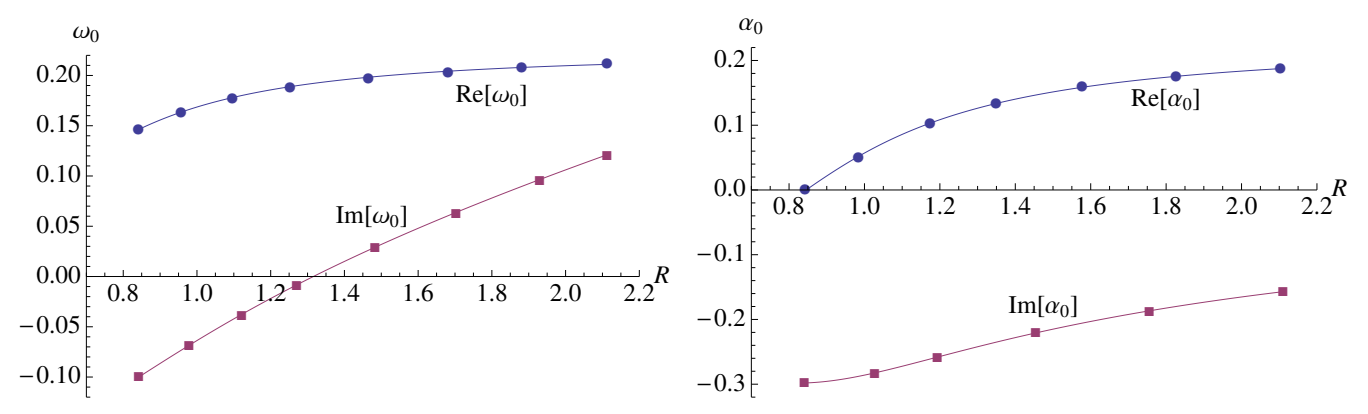

FiguRE 2. Complex frequency (left) and wavenumber (right) at the branch point as functions of the velocity ratio. Solid lines show current procedure and symbols show a few branch points extracted from Huerre \& Monkewitz (1985).
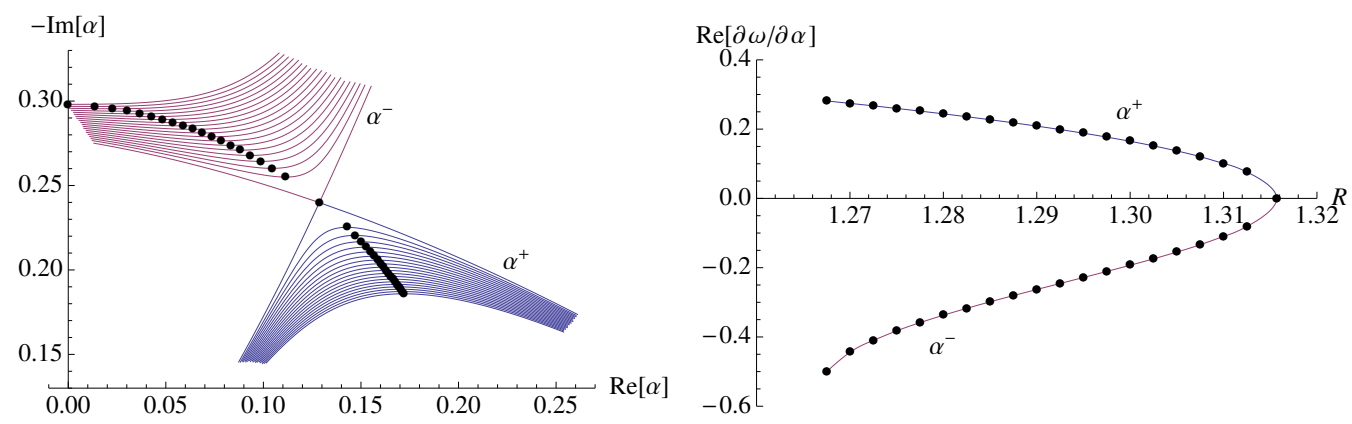

FIGURE 3. Complex streamwise wavenumber plane (left) and real group velocity (right) as functions of the velocity ratio for the planar mixing layer. Symbols in both plots represent the same modes at their respective velocity ratio.

can now be solved with Eq. (3.4) by a Newton-type root finding method to produce the two complex eigenfunctions $P_{n}(z)$ and $P_{\alpha}(z)$ as well as two complex eigenvalues.

The latter two can in fact be any two complex scalars, or four real scalars. For instance, one can search for the branch point $\operatorname{Re}\left[\alpha_{0}\right], \operatorname{Im}\left[\alpha_{0}\right], \operatorname{Re}\left[\omega_{0}\right]$ and $\operatorname{Im}\left[\omega_{0}\right]$ for a prescribed value of $R$. Figure 2 presents a comparison between these branch points calculated with the present methodology and the ones obtained by Huerre \& Monkewitz (1985) using path $D_{2}$ in Fig. 1, which were extracted from Figs. 5 and 6 of their paper. An excellent agreement can be observed. One can also search for the parametric condition $R_{0}$ at the linear onset of absolute instability instead by prescribing $\operatorname{Im}\left[\omega_{0}\right]=0$ and calculating $\operatorname{Re}\left[\alpha_{0}\right], \operatorname{Im}\left[\alpha_{0}\right], \operatorname{Re}\left[\omega_{0}\right]$ and $R_{0}$. It yields $R_{0}=1.3156935913$ and $\operatorname{Re}\left[\omega_{0}\right]=0.1921122457$. These results have an absolute error of $O\left(10^{-10}\right)$ and agree well with the values $R_{0}=$ 1.315 and $\operatorname{Re}\left[\omega_{0}\right]=0.192$ reported by Huerre \& Monkewitz (1985).

Although this validation confirms that the branch point singularities calculated here satisfy causality, a collision check is also performed and shown on the left plot of Fig. 3 . The symbols on each spatial branch involved in the collision indicate the location of the modes with a real group velocity, which is shown on the right plot of Fig. 3 as functions of $R \cdot \operatorname{Re}[\alpha], \operatorname{Im}[\alpha], \operatorname{Re}[\omega]$ and $\operatorname{Re}[\partial \omega / \partial \alpha]$ are the four scalars searched for using this methodology, while imposing $\operatorname{Im}[\omega]=0$ and $\operatorname{Im}[\partial \omega / \partial \alpha]=0$ as well as prescribing $R$. There are no more modes with these characteristics beyond the branch point, since $\operatorname{Im}[\omega]=0$ is no longer true but $\operatorname{Im}[\omega]>0$ instead. This plot clearly demonstrates that the colliding spatial modes in these branches do propagate in opposite directions before the branch point is reached, which is actually true for all modes in these branches. 
Finally, it is possible to re-write Eq. (3.2) in the form of Eq. (2.20) with $s=0$ using operator $\mathcal{P}=-1 /\left(\omega-\alpha U_{b}\right)^{2}$, which satisfies the symmetry condition $\mathcal{P} \mathcal{L}=(\mathcal{P} \mathcal{L})^{T}$. Hence, the solvability condition defined in Eq. (2.18) for this problem becomes

$$
\int_{-\infty}^{+\infty}\left\{\frac{2 \alpha P_{n}^{2}}{\left(\omega-\alpha U_{b}\right)^{2}}-\left(\omega-\alpha \frac{\partial \omega}{\partial \alpha}\right) \frac{2\left(D U_{b}\right) P_{n}\left(D P_{n}\right)}{\left(\omega-\alpha U_{b}\right)^{4}}\right\} d z=0 \quad .
$$

Re-writing Eq. (3.8) to calculate the group velocity at $R=1$, according to Eq, (2.21), yields a value of $O\left(10^{-10}\right)$, which is the same order of magnitude of the eigenvalue absolute error, confirming that the branch point calculated at $R=1$ is indeed correct.

\subsection{Pseudo Two-Dimensional Branch Point Singularities}

The second test case reproduces the pseudo two-dimensional $(\operatorname{Re}[\beta] \neq 0$ but $\operatorname{Im}[\beta]=0)$ absolute instability for a rotating disk from Lingwood (1995). This is the boundary layer flow formed on top of an infinitely wide disk as it rotates. The base state feeding the linear instability has two approximately parallel velocity components. It is an inflectional three-dimensional boundary layer, which is susceptible to an inviscid crossflow instability, similar to a swept wing. Convective instability occurs near the centerline whereas absolute instability occurs sufficiently far from it. This local base state is defined on a semi-infinite domain and obtained from the boundary layer equations

$$
\begin{gathered}
D P_{b}+W_{b} D W_{b}-D^{2} W_{b}=0 \quad \text { and } \quad 2 U_{b} V_{b}+W_{b} D V_{b}-D V_{b}=0 \\
U_{b}^{2}+D U_{b} W_{b}-V_{b}^{2}-D^{2} U_{b}=0 \quad, \quad 2 U_{b}+D W_{b}=0
\end{gathered}
$$

for the streamwise, spanwise and transverse velocity components $U_{b}(z), V_{b}(z)$ and $W_{b}(z)$, respectively, as well as pressure $P_{b}(z)$, discussed in Schlichting (1986) and subject to boundary conditions

$$
U_{b}(0)=W_{b}(0)=P_{b}(0)=0 \quad, \quad V_{b}(0)=1 \quad \text { and } \quad U_{b}(\infty)=V_{b}(\infty)=0 \quad,
$$

where the Reynolds number $R e$ is a control parameter built into the dimensionless variables. The direct differential eigenvalue problem, written in the form of Eq. (2.3) for the linear, modal and inviscid vertical velocity eigenfunction $w_{n}(z)$, is given by

$$
\mathcal{L} w_{n}=0 \quad \text { with } \quad \mathcal{L}=\left(\alpha U_{b}+\bar{\beta} V_{b}-\omega\right)\left(D^{2}-k^{2}\right)-\left(\alpha D^{2} U_{b}+\bar{\beta} D^{2} V_{b}\right)
$$

where $k^{2}=\alpha^{2}+\bar{\beta}^{2}$ and $\bar{\beta}=\beta / R e$ is the normalised pseudo spanwise wavenumber, which acts as the sole control parameter. It is subject to boundary conditions

$$
w_{n}(z=0)=0 \quad \text { and } \quad w_{n}(z \rightarrow \infty)=c_{2} e^{-\sqrt{\alpha^{2}+\bar{\beta}^{2}} z}
$$

where Eq. (3.11) is marched from infinity to the disk wall using initial conditions obtained from the right boundary condition in Eq. (3.12). In numerical terms, infinity is a high enough value of $z$ where the base state second derivatives are negligible. The Newton-type root finding method can then be used to find a solution that satisfies

$$
f_{n}(\alpha, \omega)=\tilde{w}_{n}(z=0)=0,
$$

which is the normalised version of the left boundary condition in Eq. (3.12) for the normalised disturbance $\tilde{w}_{n}(z)=w_{n}(z) / c_{2}$, avoiding the need to find constant $c_{2}$.

Taking the derivative of Eq. (3.11) with respect to $\alpha$, while noting that $\partial U_{b} / \partial \alpha=0$, 

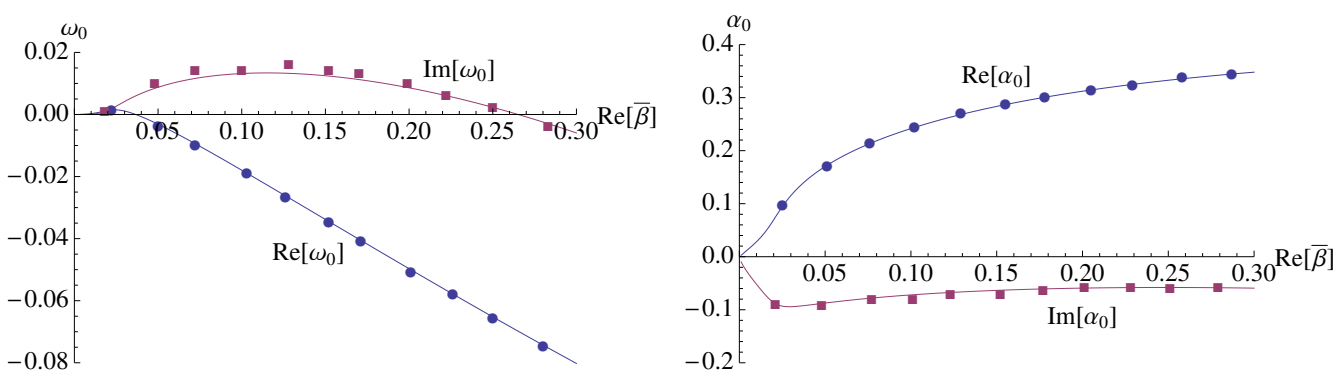

FiguRE 4. Complex frequency (left) and wavenumber (right) at the branch point as functions of the modified spanwise wavenumber. Solid lines show current procedure and symbols show a few branch points extracted from Lingwood (1995).

$\partial V_{b} / \partial \alpha=0$ and $\partial \bar{\beta} / \partial \alpha=0$, leads to the auxiliary differential eigenvalue problem

$$
\begin{gathered}
\mathcal{L} w_{\alpha}=\mathcal{S}_{\alpha}=-\mathcal{L}_{\alpha} w_{n} \text { with } \\
\mathcal{L}_{\alpha}=k^{2} \frac{\partial \omega}{\partial \alpha}+2 \alpha \omega-\left(3 \alpha^{2}+\bar{\beta}^{2}\right) U_{b}-D^{2} U_{b}-2 \alpha \bar{\beta} V_{b}-\left(\frac{\partial \omega}{\partial \alpha}-U_{b}\right) D^{2},
\end{gathered}
$$

where $w_{\alpha}(z)=\partial w_{n} / \partial \alpha$ is defined as an additional eigenfunction. This equation is subject to boundary conditions

$$
\begin{aligned}
w_{\alpha}(z=0) & =0 \text { and } \\
w_{\alpha}(z \rightarrow \infty) & =\left(c_{4}-\alpha c_{2}\left(\frac{1}{2\left(\alpha^{2}+\bar{\beta}^{2}\right)}+\frac{z}{\sqrt{\alpha^{2}+\bar{\beta}^{2}}}\right)\right) e^{-\sqrt{\alpha^{2}+\bar{\beta}^{2}} z},
\end{aligned}
$$

which can be further simplified by choosing $c_{4}=\alpha c_{2} /\left(2\left(\alpha^{2}+\bar{\beta}^{2}\right)\right)$, yielding the same boundary conditions one would obtain by taking the derivative of Eq. (3.11) with respect to $\alpha$ while neglecting the dependence of $c_{2}$ on $\alpha$. Once again, this is possible because Eqs. (3.14) and (3.15) are also an eigenvalue problem, which is undetermined up to a constant. The Newton-type method now finds a solution that satisfies Eq. (3.13) and

$$
f_{\alpha}(\alpha, \omega)=\bar{w}_{\alpha}(z=0)=0,
$$

which is the normalised left boundary condition in (3.15) for the normalised disturbance $\bar{w}_{\alpha}(z)=w_{\alpha}(z) / c_{2}$. This shooting method produces the two complex eigenfunctions $\bar{w}_{n}(z)$ and $\bar{w}_{\alpha}(z)$ as well as two complex eigenvalues, or four arbitrary real scalars.

Selecting $\operatorname{Re}\left[\alpha_{0}\right], \operatorname{Im}\left[\alpha_{0}\right], \operatorname{Re}\left[\omega_{0}\right]$ and $\operatorname{Im}\left[\omega_{0}\right]$ as the four scalars enables this methodology to search for branch points at prescribed values of $\bar{\beta}$. Figure 4 compares them with the ones obtained by Lingwood (1995) using path $D_{2}$ in Fig. 1, which were extracted from Fig. 4 of their paper. The agreement is good in most cases but less so for $\operatorname{Im}\left[\omega_{0}\right]$, which is most likely due to the poor quality of the scanned paper available online. This can be confirmed by a quantitative comparison of the branch point at $\bar{\beta}_{0}=0.126$, which yields $\omega_{0}=-0.0261664452+0.0133000530 i$ and $\alpha_{0}=0.2655922101-0.0669389400 i$ with an absolute error of $O\left(10^{-11}\right)$. These numbers agree well with the results $\omega_{0}=-0.0262+$ $0.0133 i$ and $\alpha_{0}=0.266-0.0670 i$ reported by Lingwood (1995). Imposing $\operatorname{Im}\left[\omega_{0}\right]=0$ and searching for $\bar{\beta}$ instead reveals that the onset of absolute instability occurs at $\bar{\beta}_{0}=$ $0.26514812331, \operatorname{Re}\left[\omega_{0}\right]=-0.06982182860$ and $\alpha_{0}=0.33786742527-0.05817350830 i$ with the same absolute error. These numbers agree well with the values $\bar{\beta}_{0}=0.265$, $\operatorname{Re}\left[\omega_{0}\right]=-0.0698$ and $\alpha_{0}=0.338-0.0582 i$, also reported by Lingwood (1995).

Once again, it is important to verify the collision criterion in order to demonstrate 

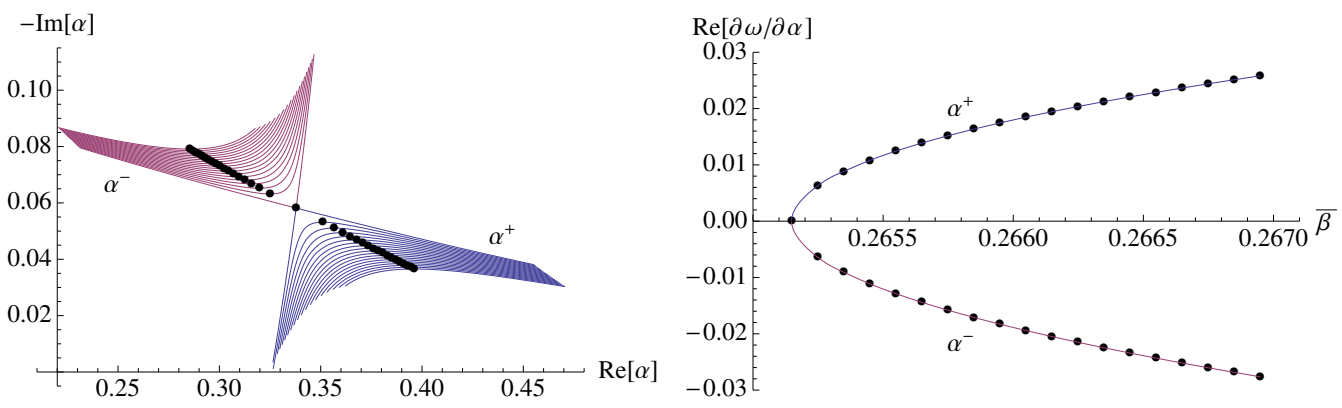

Figure 5. Complex streamwise wavenumber plane (left) and real group velocity (right) as functions of the normalised spanwise wavenumber for the rotating disk. Symbols in both plots represent the same modes at their respective normalised spanwise wavenumber.

causality and show its relation to the group velocity. Figure 5 shows the collision check within the convectively unstable region up to the onset of absolute instability on the left plot, where the symbols indicate the location of the mode with a real group velocity on each spatial branch involved in the collision. These same symbols are also shown on the right plot of the same figure, which presents their real group velocity as a function of the normalised spanwise wavenumber. They were calculated by selecting $\operatorname{Re}[\alpha], \operatorname{Im}[\alpha], \operatorname{Re}[\omega]$ and $\operatorname{Re}[\partial \omega / \partial \alpha]$ as the four scalars searched for using this methodology, while imposing $\operatorname{Im}[\omega]=0$ and $\operatorname{Im}[\partial \omega / \partial \alpha]=0$ as well as prescribing $\bar{\beta}$. This is the reason why no more modes with these characteristics can be included beyond the branch point singularity, since $\operatorname{Im}[\omega]=0$ is no longer true but $\operatorname{Im}[\omega]>0$ instead. Once again, these modes in their respective colliding spatial branches do propagate in opposite directions before the branch point is reached.

Finally, it is possible to re-write Eq. (3.11) in the form of Eq. (2.20) with $s=0$ using operator $\mathcal{P}=1 /\left(\omega-\alpha U_{b}-\bar{\beta} V_{b}\right)$, which satisfies the symmetry condition $\mathcal{P} \mathcal{L}=(\mathcal{P} \mathcal{L})^{T}$. Hence, the solvability condition in Eq. (2.18) for this problem becomes

$$
\begin{gathered}
\int_{0}^{\infty}\left\{\left(\frac{\partial \omega}{\partial \alpha}-U_{b}\right) \frac{w_{n} D^{2} w_{n}}{\omega-\alpha U_{b}-\bar{\beta} V_{b}}-\left(k^{2} \frac{\partial \omega}{\partial \alpha}+2 \alpha \omega\right.\right. \\
\left.\left.-\left(3 \alpha^{2}+\bar{\beta}^{2}\right) U_{b}-D^{2} U_{b}-2 \alpha \bar{\beta} V_{b}\right) \frac{w_{n}^{2}}{\omega-\alpha U_{b}-\bar{\beta} V_{b}}\right\} d z=0 .
\end{gathered}
$$

Following Eq. (2.21), the group velocity at $\bar{\beta}=0.126$ is extracted from Eq. (3.17) to yield a value of $O\left(10^{-6}\right)$, which is five orders of magnitude higher than the eigenvalue absolute error. This integrand decays slowly and, hence, its non-negligible contributions extend far into the $z$ domain. Error cumulates because it becomes increasingly difficult to generate accurate base flow solutions for such large $z$ values.

\subsection{Three-Dimensional Branch Point Singularities}

The third test case is the three-dimensional $(\alpha \neq 0$ and $\beta \neq 0)$ absolute instability of the heated flow in a saturated homogeneous porous medium reported by Brevdo (2009). This is the flow generated by an inclined temperature gradient and a vertical throughflow. The base state has a non-parallel but still local two-component velocity field and a non-local two-dimensional temperature profile. This forced base flow convection can be superposed with natural convection due to buoyancy driven instabilities, which can be either convective or absolute, depending on a non-trivial combination of the three 
governing parameter values. Its local base state is given by

$$
\begin{gathered}
U_{b}(z)=R_{h} z \quad, \quad V_{b}(z)=0 \quad, \quad W_{b}(z)=Q_{v} \quad \text { and } \\
T_{b}(z)=\frac{R_{h}^{2}}{2 Q_{v}}\left(z^{2}+\frac{2 z}{Q_{v}}-\frac{1}{4}\right)-\frac{Q_{v}^{2} R_{v}+R_{h}^{2}}{2 Q_{v}^{2} \sinh \left[Q_{v} / 2\right]}\left(e^{Q_{v} z}-\cosh \left[Q_{v} / 2\right]\right)-R_{h} x,
\end{gathered}
$$

for the streamwise, spanwise and transverse velocity components $U_{b}(z), V_{b}(z)$ and $W_{b}(z)$, respectively, as well as temperature $T_{b}(z)$, defined on the finite domain $-1 / 2 \leqslant z \leqslant+1 / 2$. $R_{v}, R_{h}$ and $Q_{v}$ are the vertical and horizontal Rayleigh numbers and Péclet number, respectively. The direct differential eigenvalue problem, written in the form of Eq. (2.3) for the linear and modal transverse velocity eigenfunction $w_{n}(z)$, is given by

$$
\begin{aligned}
\mathcal{L} w_{n}=0 \quad \text { with } \quad \mathcal{L} & =D^{4}-Q_{v} D^{3}-\left(2 k^{2}-i\left(\omega-\alpha U_{b}\right)\right) D^{2}+\left(Q_{v} k^{2}-i \alpha R_{h}\right) D \\
& +k^{2}\left(k^{2}-i\left(\omega-\alpha U_{b}\right)+D T_{b}\right)
\end{aligned}
$$

where $k^{2}=\alpha^{2}+\beta^{2}$ and the $x$ dependence of the base flow temperature is omitted, i.e. $T_{b}(z)$ is used instead of $T_{b}(x, z)$, since only a derivative of $T_{b}$ with respect to $z$ appears in Eq. (3.19). This equation is subject to boundary conditions

$$
w_{n}(z= \pm 1 / 2)=D^{2} w_{n}(z= \pm 1 / 2)=0,
$$

and marched from $z=-1 / 2$ to $+1 / 2$ using as initial conditions the boundary conditions at $z=-1 / 2$ in Eq. (3.20), in addition to

$$
D w_{n}(z=-1 / 2)=c_{1} \text { and } D^{3} w_{n}(z=-1 / 2)=c_{2} \quad,
$$

where $c_{1}$ and $c_{2}$ are unknown complex constants. The Newton-type root finding method can then be used to find a solution that satisfies

$$
f_{n}(\alpha, \beta, \omega)=\left\{\tilde{w}_{n}(z=+/ 2), D^{2} \tilde{w}_{n}(z=+1 / 2)\right\}=0,
$$

which is the normalised version of the boundary conditions at $z=+1 / 2$ in Eq. (3.20) for the normalised disturbance $\tilde{w}_{n}(z)=w_{n}(z) / c_{2}$, avoiding the need to find constant $c_{2}$. Contrary to the previous two test cases, $f_{n}(\alpha, \beta, \omega)=0$ represents two complex equations for a single complex eigenvector, namely $w_{n}(z)$, but two complex eigenvalues. In this particular case, $c_{1}$ is also an eigenvalue and it must be obtained. Hence, this allows the search for only one physically meaningful complex eigenvalue, or two real scalars.

Taking the derivative of Eq. (3.19) with respect to $\alpha$, while noting that $\partial U_{b} / \partial \alpha=$ $\partial T_{b} / \partial \alpha=0$ and $\partial R_{h} / \partial \alpha=\partial R_{v} / \partial \alpha=\partial Q_{v} / \partial \alpha=0$, leads to the auxiliary problem

$$
\begin{gathered}
\mathcal{L} w_{\alpha}=\mathcal{S}_{\alpha}=-\mathcal{L}_{\alpha} w_{n} \text { with } \\
\mathcal{L}_{\alpha}=2 \alpha\left(2 k^{2}-i\left(\omega-\alpha U_{b}\right)+D T_{b}\right)-i k^{2}\left(\frac{d \omega}{d \alpha}-U_{b}\right) \\
+\left(2 Q_{v} \alpha-i R_{h}\right) D-\left(4 \alpha-i\left(\frac{d \omega}{d \alpha}-U_{b}\right)\right) D^{2}
\end{gathered}
$$

where $w_{\alpha}(z)=\partial w_{n} / \partial \alpha$ is defined as an additional eigenfunction. This equation is subject to boundary conditions

$$
w_{\alpha}(z= \pm 1 / 2)=D^{2} w_{\alpha}(z= \pm 1 / 2)=0,
$$

obtained by taking the derivative of Eq. (3.20) with respect to $\alpha$. In order to transform Eqs. (3.23) to (3.24) into an initial value problem, the additional initial conditions

$$
D w_{\alpha}(z=-1 / 2)=c_{3} \text { and } D^{3} w_{\alpha}(z=-1 / 2)=c_{4} \quad,
$$


are employed, as done with Eq. (3.21), where $c_{3}$ and $c_{4}$ are unknown complex constants. The root finding method employed previously now must solve Eq. (3.22) and

$$
f_{\alpha}(\alpha, \beta, \omega)=\left\{\tilde{w}_{\alpha}(z=+/ 2), D^{2} \tilde{w}_{\alpha}(z=+1 / 2)\right\}=0,
$$

which is the normalised version of the boundary conditions at $z=+1 / 2$ in Eq. (3.24) for the normalised disturbance $\tilde{w}_{\alpha}(z)=w_{\alpha}(z) / c_{4}$, avoiding the need to find constant $c_{4}$. Equations (3.22) and (3.26) yield four complex equations for two complex eigenvectors, namely $w_{n}(z)$ and $w_{\alpha}(z)$, as well as four complex eigenvalues. Now, $c_{1}$ and $c_{3}$ are also eigenvalues and must be obtained. Hence, this allows the search for two physically meaningful complex eigenvalues, or four real scalars.

Up to this point, one can use the above equations to solve either a two-dimensional or a pseudo two-dimensional linear and local stability problem by prescribing $\beta=0$ or $\operatorname{Re}[\beta] \neq 0$ and $\operatorname{Im}[\beta]=0$, respectively. In order to solve a three-dimensional stability problem instead, the value of $\beta$ must not be prescribed a priori but obtained as a solution as well. Hence, an additional auxiliary equation is required. Taking the derivative of Eq. (3.19) with respect to $\beta$, while noting that $\partial U_{b} / \partial \beta=\partial T_{b} / \partial \beta=0$ and $\partial R_{h} / \partial \beta=$ $\partial R_{v} / \partial \beta=\partial Q_{v} / \partial \beta=0$, leads to additional auxiliary differential eigenvalue problem

$$
\begin{gathered}
\mathcal{L} w_{\beta}=\mathcal{S}_{\beta}=-\mathcal{L}_{\beta} w_{n} \text { with } \\
\mathcal{L}_{\beta}=2 \beta\left(2 k^{2}-i\left(\omega-\alpha U_{b}\right)+D T_{b}\right)-i k^{2} \frac{d \omega}{d \beta}+2 Q_{v} \beta D-\left(4 \beta-i \frac{d \omega}{d \beta}\right) D^{2},
\end{gathered}
$$

where $w_{\beta}(z)=\partial w_{n} / \partial \beta$ is defined as an additional eigenfunction. This equation is subject to boundary conditions

$$
w_{\beta}(z= \pm 1 / 2)=D^{2} w_{\beta}(z= \pm 1 / 2)=0,
$$

obtained by taking the derivative of Eq. (3.20) with respect to $\beta$. In order to transform Eqs. (3.27) to (3.28) into an initial value problem, the additional initial conditions

$$
D w_{\beta}(z=-1 / 2)=c_{5} \text { and } \quad D^{3} w_{\beta}(z=-1 / 2)=c_{6} \quad,
$$

are employed, as done with Eqs. (3.21) and (3.25), where $c_{5}$ and $c_{6}$ are unknown complex constants. The root finding method is now adapted to solve Eqs. (3.22), (3.26) and

$$
f_{\beta}(\alpha, \beta, \omega)=\left\{\tilde{w}_{\alpha}(z=+/ 2), D^{2} \tilde{w}_{\beta}(z=+1 / 2)\right\}=0,
$$

which is the normalised version of the boundary conditions at $z=+1 / 2$ in Eq. (3.28) for the normalised disturbance $\tilde{w}_{\beta}(z)=w_{\beta}(z) / c_{6}$, avoiding the need to find constant $c_{6}$. Equations (3.22), (3.26) and (3.30) represent six complex equations for three complex eigenvectors, namely $w_{n}(z), w_{\alpha}(z)$ and $w_{\beta}(z)$, as well as six complex eigenvalues. In this case, $c_{1}, c_{3}$ and $c_{5}$ are also eigenvalues that must be obtained. Hence, this allows the search for three physically meaningful complex eigenvalues, or six real scalars.

Figure 6 presents results at the onset of convective instability obtained by the current procedure for different values of the Péclet and horizontal Rayleigh numbers, comparing them with the results in Tabs. 1 and 3 from Brevdo (2009). Solid lines represent real group velocities (left) and critical vertical Rayleigh numbers (right). The former was obtained by selecting $\operatorname{Re}[\alpha], \operatorname{Re}[\beta], \operatorname{Re}[\omega], R_{v}, \operatorname{Re}[\partial \omega / \partial \alpha]$ and $\operatorname{Re}[\partial \omega / \partial \beta]$ as the six real scalars provided by the method while imposing $\operatorname{Im}[\alpha]=\operatorname{Im}[\beta]=\operatorname{Im}[\omega]=0$ and $\operatorname{Im}[\partial \omega / \partial \alpha]=\operatorname{Im}[\partial \omega / \partial \beta]=0$ for prescribed values of $R_{h}$ and $Q_{v}$. On the other hand, the latter was obtained by selecting $\operatorname{Re}\left[\alpha_{0}\right], \operatorname{Im}\left[\alpha_{0}\right], \operatorname{Re}\left[\beta_{0}\right], \operatorname{Re}\left[\beta_{0}\right], \operatorname{Re}[\omega]$ and $R_{v}$ as the six real scalars provided by the method while imposing $\operatorname{Im}\left[\omega_{0}\right]=0$ and $\partial \omega / \partial \alpha=$ $\partial \omega / \partial \beta=0$ for prescribed values of $R_{h}$ and $Q_{v}$. Brevdo (2009) calculates the onset of 

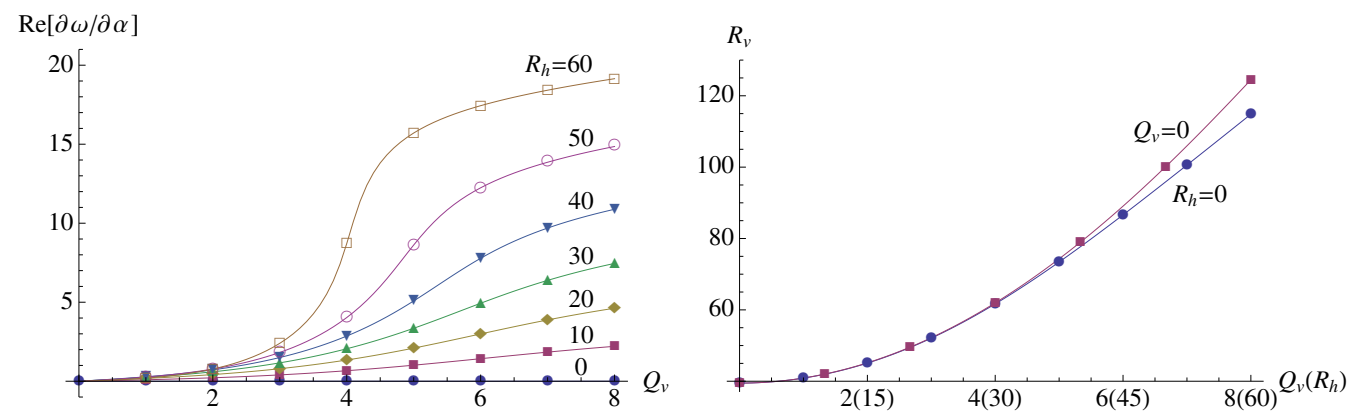

FiguRE 6. Real group velocity (left) and vertical Rayleigh number (right) at the branch point as functions of the Péclet number (left and right) or horizontal Rayleigh number (right). Cases where $Q_{v} R_{h}=0$ satisfy causality. $\operatorname{Re}[\partial \omega / \partial \beta]=0$ for all cases. Solid lines show current procedure and symbols show a few data points taken from Brevdo (2009).

convective instability using path $D_{1}$ in Fig. 1 and then evaluates the group velocity at these conditions in order to check if they might represent an onset of absolute instability as well. The former figure confirms his finding that the onset of convective and absolute instability only coincide when $Q_{v} R_{h}=0$, since the group velocity only goes to zero when either $Q_{v}=0$ or $R_{h}=0$. Critical vertical Rayleigh numbers for the onset of absolute instability at these two limiting cases are shown in the right figure, agreeing with his calculations where the onset of convective and absolute instability coincide. These numbers at $Q_{v}=8$ and $R_{h}=0$ as well as $Q_{v}=0$ and $R_{h}=60$ are $R_{h}=$ 114.832605711515 and 124.4728454361939 , respectively obtained with as absolute error of $O\left(10^{-12}\right)$. They are in quantitative agreement with the values $R_{h}=114.84$ and 124.48 reported by Brevdo (2009) at the same parametric conditions. It is worth pointing out that the current procedure was also used to calculate the two-dimensional $(\beta=0)$ onset of absolute instability for this problem. Although these results are not shown here, they agree with Brevdo \& Ruderman (2009), showing that both convective and absolute onsets of instability coincide even when $Q_{v} R_{h} \neq 0$.

In order to provide evidence that the branch points calculated satisfy causality, a collision check was performed for a few arbitrarily chosen parametric conditions. One of them, where $Q_{v}=0$ and $R_{h}=40$, is shown in Fig. 7 (left) as a function of $R_{v}$ for both streamwise (top) and transversal (bottom) complex wavenumber planes. The former is plotted at $\beta=\beta_{0}=3.215215718763$ whereas the latter is plotted at $\alpha=\alpha_{0}=0$, which is the branch point for $R_{v}=78.96639607652$. Once again, these results were obtained with an absolute error of $O\left(10^{-12}\right)$. Symbols indicate the modes within each branch that have a real group velocity, which is shown in Fig. 7 (right). Top and bottom real group velocities shown are obtained by pseudo two-dimensional calculations imposing $\beta=\beta_{0}$ and $\alpha=\alpha_{0}$, respectively. Brevdo (2009) reported zero group velocities at $\alpha_{0}=0$, $\beta_{0}=3.22$ and $R_{v}=78.967$, in agreement with the present results. This figure indicates a collision between branches with opposite group velocities coming from opposite sides of the complex plane for both streamwise and transversal wavenumbers, confirming that the branch point singularity satisfies causality according to Brevdo (1991).

Finally, solvability conditions are evaluated for this test case. Unfortunately, it is not possible to define an operator $\mathcal{P}$ that allows the linear and homogeneous operator in Eq. (3.19) to satisfy Eq. (2.20), i.e. $\mathcal{P} \mathcal{L} \neq(\mathcal{P} \mathcal{L})^{T}$. Hence, the classical adjoint based solvability conditions defined in Eqs. (2.13) and (2.14) must be used instead of Eqs. (2.18) and (2.19), which are modified solvability conditions defined for symmetric operators. An 


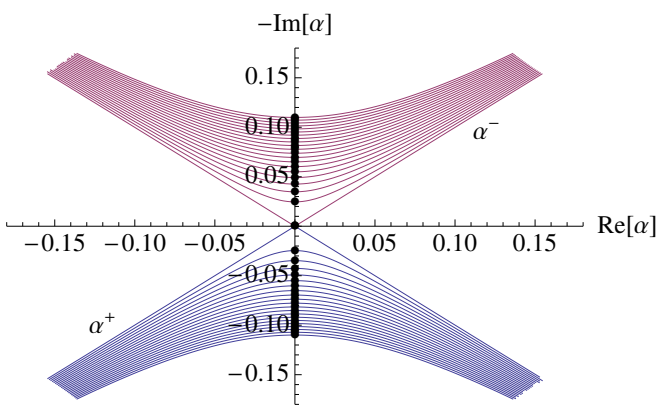

$\operatorname{Re}[\partial \omega / \partial \alpha]$
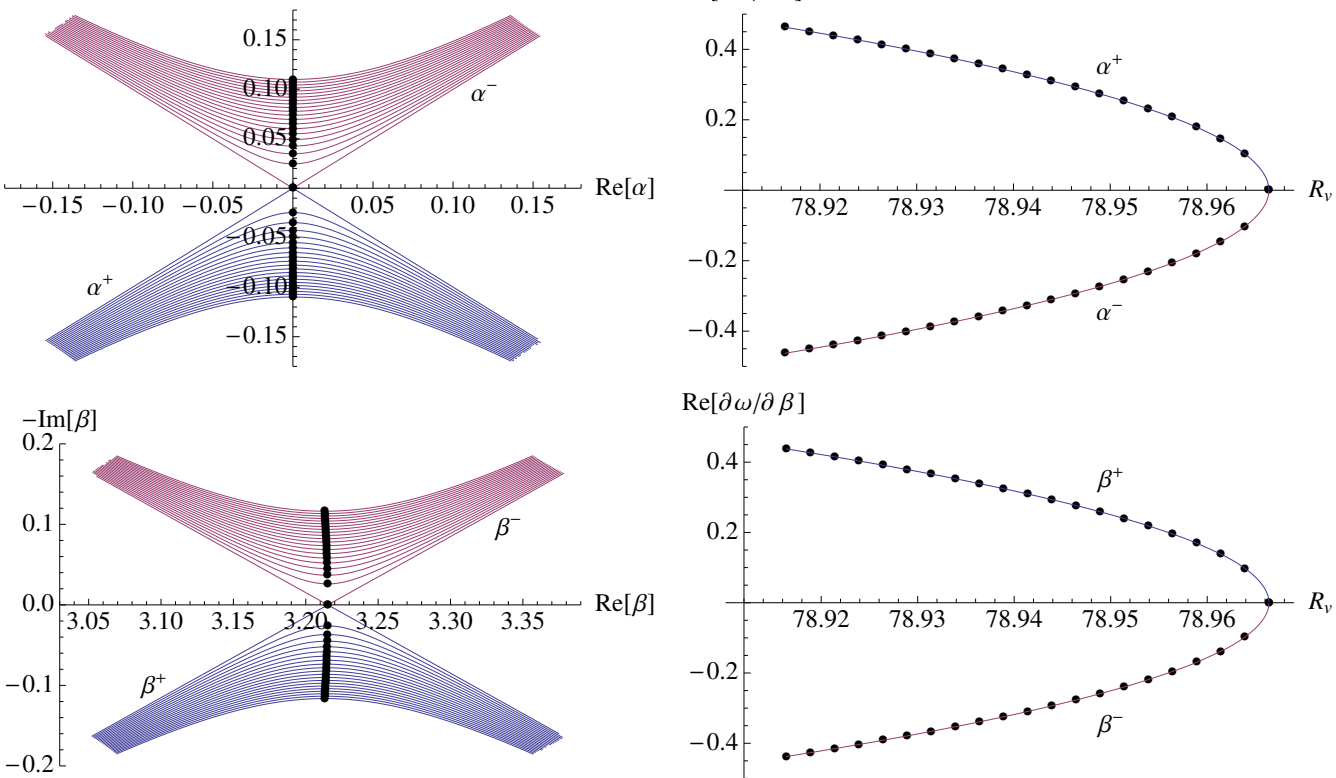

$\operatorname{Re}[\partial \omega / \partial \beta]$

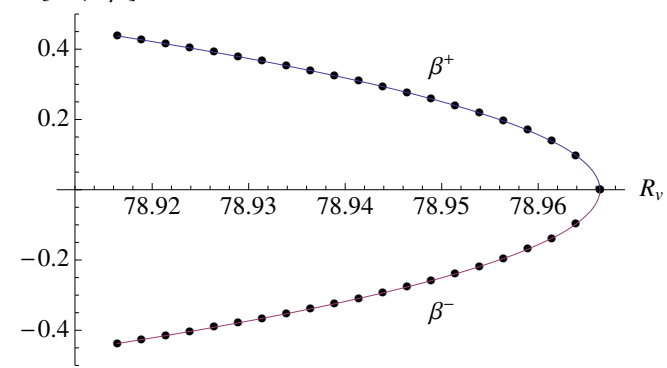

Figure 7. Complex (top) streamwise and (bottom) transverse (left) wavenumber planes as well as (top) streamwise and (bottom) transversal (right) real group velocities as functions of the vertical Rayleigh number for the porous medium problem with $Q_{v}=0$ and $R_{h}=40$. Symbols in all plots represent the same modes at their respective vertical Rayleigh number.

adjoint problem of Eq. (3.19), based on Eq. (2.12), can be written as

$$
\begin{aligned}
\mathcal{L}^{*} w_{n}^{+}=0 \quad \text { with } \quad \mathcal{L}^{*} & =D^{4}+Q_{v} D^{3}-\left(2 \bar{k}^{2}+i\left(\bar{\omega}-\bar{\alpha} U_{b}\right)\right) D^{2}-\left(Q_{v} \bar{k}^{2}-i \bar{\alpha} R_{h}\right) D \\
& +\bar{k}^{2}\left(\bar{k}^{2}+i\left(\bar{\omega}-\bar{\alpha} U_{b}\right)+D T_{b}\right)
\end{aligned}
$$

when subject to boundary conditions

$$
w_{n}^{+}(z= \pm 1 / 2)=\left(D^{2}+Q_{v} D\right) w_{n}^{+}(z= \pm 1 / 2)=0 \quad,
$$

allowing $w_{n}^{+}$to be an adjoint of $w_{n}$. The classical solvability conditions then become

$$
\begin{gathered}
\int_{-1 / 2}^{+1 / 2}\left\{\left(4 \alpha-i\left(\frac{d \omega}{d \alpha}-U_{b}\right)\right) D^{2} w_{n}-\left(2 Q_{v} \alpha-i R_{h}\right) D w_{n}\right. \\
\left.-\left(2 \alpha\left(2 k^{2}-i\left(\omega-\alpha U_{b}\right)+D T_{b}\right)-i k^{2}\left(\frac{d \omega}{d \alpha}-U_{b}\right)\right) w_{n}\right\} \bar{w}_{n}^{+} d z=0 \text { and } \\
\int_{-1 / 2}^{+1 / 2}\left\{\left(4 \beta-i \frac{d \omega}{d \beta}\right) D^{2} w_{n}-2 Q_{v} \beta D w_{n}\right. \\
\left.-\left(2 \beta\left(2 k^{2}-i\left(\omega-\alpha U_{b}\right)+D T_{b}\right)-i k^{2} \frac{d \omega}{d \beta}\right) w_{n}\right\} \bar{w}_{n}^{+} d z=0
\end{gathered}
$$

respectively. Based on Eqs. (2.15) and (2.16), the above equations can be re-written to provide formulas for their respective group velocities. They yield values of $O\left(10^{-12}\right)$ at $Q_{v}=0$ and $R_{h}=40$, which is the same order of magnitude of the eigenvalue absolute error. This confirms that the branch point calculated at $Q_{v}=0$ and $R_{h}=40$ is correct and it does allow group velocity calculations for all modes in the colliding branches. 


\subsection{Comparative Efficiency Evaluation}

It is important to evaluate the efficiency of the present methodology relative to the other traditional methods from the literature illustrated in Fig. 1. Such a comparison is very often strongly problem dependent and usually has a high degree of subjectivity, which arises from the fact that one can choose from a wide array of error tolerances, numerical schemes, software libraries, computer hardwares and so on. Hence, definitive answers are beyond the scope of this paper. It is possible, however, to provide some reasonable efficiency estimates based on different metrics. They are provided in this subsection for both two and three-dimensional absolute instabilities found in the third test case of this paper, originally studied by Brevdo (2009).

Comparisons with path A from Fig. 1 are excluded from this study. Such comparisons are not pertinent because the present methodology was developed for eigenvalue problems whose eigenfunction dependence on the single inhomogeneous coordinate cannot be written in a simple analytical form. In other words, the present methodology was developed for differential eigenvalue problems. When this analytical dependence exists, the auxiliary differential eigenvalue problems generated by this methodology naturally reduce to the same auxiliary dispersion relations one would obtain through path $\mathrm{A}$. This is implied by the proof in section 2.4, but for the particular case where $L$ is diagonal.

Matrix forming the direct differential eigenvalue problem, forcing its determinant to be zero to generate the direct dispersion relation and taking its derivative with respect to the wavenumbers to generate the auxiliary dispersion relations in order to locate branch points, as illustrated by path B in Fig. 1, can be done in one of two ways: analytically or numerically. Both were evaluated and the former is significantly less efficient than the latter. Hence, only the latter will be shown here. Matrix forming of Eq. (3.19) was achieved using series expansion $w_{n}(z)=\sum_{m=1}^{\infty} \bar{w}_{m} \psi_{m}(z)$, which employs the normalized basis function $\psi_{m}(z)=-\sqrt{2} \sin \left[\lambda_{m}(z+1 / 2)\right]$ and $\lambda_{m}=m \pi$ to satisfy all boundary conditions in Eq. (3.20). Since these basis functions are orthogonal to each other, i.e. $\int_{-1 / 2}^{+1 / 2} \psi_{m}(z) \psi_{n}(z) d z=\delta_{m, n}$, where $\delta_{m, n}$ is the Kronecker delta, the unknown coefficients in this orthogonal series expansion must satisfy $\bar{w}_{m}=\int_{-1 / 2}^{+1 / 2} \psi_{m}(z) w_{n}(z) d z$. Hence, this expansion can be substituted into Eq. (3.19), which is then multiplied by $\psi_{n}(z)$ and integrated over the domain size to yield the system of equations $\sum_{m=1}^{\infty} A_{m, n} \bar{w}_{n}=0$ for all positive $n$. Although all $A_{m, n}$ integral coefficients of matrix $A$ can be obtained analytically, only their numerical values are used when evaluating $\operatorname{det}[A]$. Its derivatives with respect to the wavenumbers are approximated with a first order finite difference discretization, i.e. $\partial \operatorname{det}[A] / \partial \alpha=(\operatorname{det}[A(\alpha+\delta \alpha)]-\operatorname{det}[A(\alpha)]) / \delta \alpha+O(\delta \alpha)$, where $\delta \alpha$ can be made as small as necessary to satisfy the user prescribed error tolerances. The same is true for $\partial \operatorname{det}[A] / \partial \beta$ and $\delta \beta$ when performing three-dimensional calculations. In addition, the same Mathematica built-in root-finding solver FindRoot employed for the new methodology was used here to solve the direct dispersion relation and these auxiliary ones when searching for branch points.

A wide range of continuation techniques to search for branch points can be found in the literature, all based on the numerical evaluation of the group velocity using frequency and wavenumber data obtained from the eigenvalue problem. This data can be generated by solving either algebraic eigenvalue problems with eigenvalue solvers or differential eigenvalue problems with shooting methods, illustrated by paths $C_{1}$ and $C_{2}$ in Fig. 1 , respectively, which can be done in the context of an either temporal or spatial stability analysis. This technique is usually expensive, since it relies on plotting group velocity planes over a complex frequency (or wavenumber) space to find where it changes sign. A much less expensive variation was developed by Suslov (2006), in which a numerical 

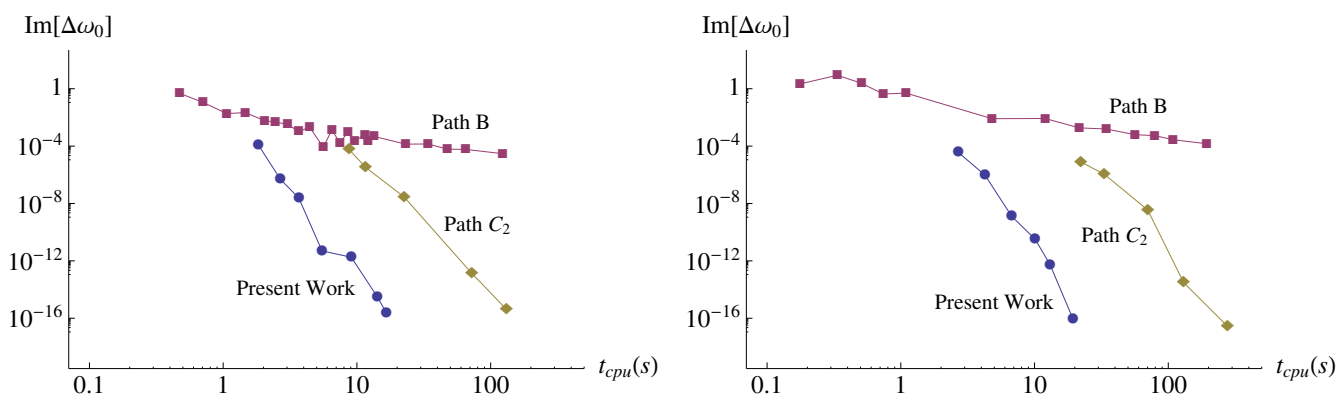

FiguRE 8. Branch point temporal growth rate absolute error versus mean computational time required by the three different methods tested for (left) two and (right) three-dimensional simulations of the problem studied by Brevdo (2009). Parametric conditions are (left) $R_{h}=30$, $Q_{v}=4$ and $R_{v}=122$ as well as (right) $R_{h}=50, Q_{v}=5$ and $R_{v}=134$.

zero group velocity equation is used as an auxiliary differential eigenvalue problem to be solved with the direct differential eigenvalue problem in the search for branch points. The auxiliary problem employed here was based on the finite difference approximation $\partial \omega / \partial \alpha=(\omega(\alpha+\delta \alpha)-\omega(\alpha)) / \delta \alpha+O(\delta \alpha)=0$, where $\delta \alpha$ can be made as small as necessary to satisfy the user prescribed error tolerances. An equivalent formula was used for $\partial \omega / \partial \beta$ when performing three-dimensional calculations. In order to further improve the efficiency of this numerical group velocity calculation, the shooting method developed to solve the direct differential eigenvalue problem for the new methodology was adapted to provide the complex frequency for a prescribed real (or complex) wavenumber. This efficiency comes from the fact that the eigenvalue spectra is not dense and good initial guesses are available. The use of matrix forming and eigenvalue solvers instead does not requires initial guesses but would provide a large number of eigenvalues, where only one would be necessary for the calculation of these numerical group velocities. Furthermore, the same root-finding solver FindRoot used for the new methodology was adapted to search for branch points using the direct problem and these auxiliary ones.

Branch point temporal growth rates were obtained using the new method and these two representative methods from paths $\mathrm{B}$ and $C_{2}$. Two-dimensional oscillatory transverse modes $\left(\beta_{0}=0\right)$ were obtained for $R_{h}=30, Q_{v}=4$ and $R_{v}=122$ using as initial guesses for their respective branch point searches $\operatorname{Re}\left[\alpha_{0}\right]=5.5, \operatorname{Im}\left[\alpha_{0}\right]=-3.3, \operatorname{Re}\left[\omega_{0}\right]=3.39$ and $\operatorname{Im}\left[\omega_{0}\right]=0.0$. On the other hand, three-dimensional stationary longitudinal modes were obtained for $R_{h}=50, Q_{v}=5$ and $R_{v}=134$ using as initial guesses $\operatorname{Re}\left[\alpha_{0}\right]=0.0$, $\operatorname{Im}\left[\alpha_{0}\right]=-0.6, \operatorname{Re}\left[\beta_{0}\right]=4.0, \operatorname{Im}\left[\beta_{0}\right]=0.0, \operatorname{Re}\left[\omega_{0}\right]=0.0$ and $\operatorname{Im}\left[\omega_{0}\right]=0.0$. All methods generated the same branch point in each case, which cross-verified their own results. Their respective absolute errors were estimated using the most accurate solution obtained from each method. All error control parameters for each method, e.g. both WorkingPrecision in the new method as well as $\delta \alpha$ and $\delta \beta$ in the two representative methods from paths $\mathrm{B}$ and $C_{2}$, were carefully adjusted so each target error tolerance was achieved within the smallest possible computational time. Mean computational times were generated by averaging data from 20 simulations at each parametric condition. Percentage standard deviations for the new method and the presentative methods from paths $\mathrm{B}$ and $C_{2}$ respectively remained between $[0.19,2.07],[0.19,10.2]$ and $[0.59,4.10]$ for the two-dimensional case and $[1.65,8.15],[0.27,9.40]$ and $[1.31,3.56]$ for the threedimensional one, with the smallest (largest) values at the largest (smallest) times. These results are shown in Fig. (8) for both (left) two and (right) three-dimensional branch points. Branch point frequency, wavenumber and spatial growth rate absolute errors 
have the same trends and, hence, are not shown. As expected, the path $\mathrm{B}$ method is the least efficient one when accurate solutions are sought. However, it is the most efficient one to provide very low/low accuracy estimations. It was in fact also used for this very purpose by Brevdo (1995), Carrière \& Monkewitz (1999) and Hirata et al. (2015). The representative path $C_{2}$ method and the new method can provide equivalent low/medium to high accuracy solutions, but the former is approximately one order of magnitude slower than the latter for any given target error tolerance in both two and three-dimensional problems. Furthermore, the efficiency loss when searching for singularities in three-dimensional problems instead of two-dimensional ones is much higher for the representative path $C_{2}$ method than for the new method. For instance, the mean computational time required to reach a solution with $10^{-5}\left(10^{-10}\right)$ absolute error is $133 \%$ (110\%) higher for the former but only $41.6 \%$ (58.6\%) higher for the latter. Hence, enforcing the zero group velocity conditions implicitly through auxiliary equations derived from the eigenvalue problem, as the new method does for differential eigenvalue problems and path A methods traditionally do for dispersion relations, leads to a more efficient methodology than explicitly using the zero velocity conditions themselves as auxiliary equations, as the representative path $C_{2}$ method does. This result also provides indirect evidence that solving Eq. (2.3) with Eqs. (2.8) and (2.9) should be a lot more efficient than doing so explicitly using group velocities obtained from Eqs. (2.15) and (2.16) or even with Eqs. (2.21) and (2.22).

Branch point detection through direct collision checks, as illustrated in paths D from Fig. 1, is arguably the most inefficient procedure and, hence, was not included in the above efficiency comparison analysis. It should only be used to prove causality, not to search for branch point singularities. This inefficiency, however, can be demonstrated using operation counts in two and three-dimensional branch point calculations for a single control parameter set based on the new methodology as well as a direct collision check. The latter can be performed by solving for $\alpha$ using Eq. (2.3) at $N$ values of $\operatorname{Re}[\omega]$ and another $N$ values of $\operatorname{Im}[\omega]$. Assuming the number of iterations required by the shooting method for each solve is $O(n)$, the total number of iterations in a collision check would be $O\left(n \times N^{2}\right)$. On the other hand, the former solves the coupled system given by Eqs. (2.3) and (2.8) for $\alpha$ and $\omega$ with the same shooting method, which requires approximately $O(2 \times n)$ iterations. Extending this reasoning to three-dimensional branch point calculations, the total number of iterations would become $O\left(2 \times n^{2} \times N^{2}\right)$ and $O(3 \times n)$, respectively. If one assumes $n=N=10$ for the sake of simplicity, the new method would require two and three orders of magnitude less iterations than the collision check for two and three-dimensional branch points calculations, respectively. Since $N \gg$ $n$, usually, this cost difference can be much higher.

\section{Conclusions}

The theoretical concepts related to convective and absolute instabilities of local or nearly local base flows have been known for several decades. However, the numerical costs associated with the different methods used to identify branch point singularities in differential eigenvalue problems makes this analysis still quite cumbersome when these problems take the form of ordinary differential equations. What is known about these costs (see, for instance, Suslov 2006) can be summarized as follows:

(i) A direct causality proof requires deforming the integrations contours that define the linear impulse response into a steepest descent path that contains the branch point singularity. However, deforming these integration lines for wave packets that propagate in one direction (named here a two-dimensional problem) is quite expensive. Doing so 
for integration surfaces when the wave packet propagates in two directions (named here three-dimensional problem) is essentially prohibitive.

(ii) The first popular alternative that was proposed early on, to search for branch point singularities and demonstrate causality, is known as the collision criterion. The most common version graphically verifies that saddle points in a complex wavenumber plane are formed by the collision between downstream and upstream propagating branches that came from opposite sides of this complex plane. This graphical verification can be performed on a complex frequency plane as well, but one observes the formation of cusp points instead. Either approach is feasible, although still expensive, for two-dimensional problems. However, extensions to three-dimensional problems are still prohibitive.

(iii) When the eigenfunction dependence on the single inhomogeneous coordinate has an analytical form, the differential eigenvalue problem reduces to a dispersion relation, which represents an algebraic relationship between the eigenvalues. Under this scenario, it is straightforward to take derivatives of the dispersion relation with respect to the wavenumbers, apply the zero group velocity conditions and generate auxiliary dispersion relations. Solving these coupled direct and auxiliary dispersion relations with root-finding methods enables one to efficiently search for branch point singularities under a wide range of parametric conditions. Once these branch points are known, the collision criterion can be applied more efficiently to verify causality. In order to further minimize computational times, this is done only for a small parametric sample size, made large enough to generate sufficient confidence that the entire set satisfies causality. Both two and three-dimensional problems can be efficiently solved using this methotolody.

(iv) However, the eigenfunction dependence on the single inhomogeneous coordinate does not have an analytical form in general. In these cases, the resulting eigenvalue problem remains an ordinary differential equation and the eigenfunction must be obtained numerically. Two alternative methods are then commonly used:

(a) Matrix forming is applied to the differential eigenvalue problem, usually based on a spectral method, transforming it into an algebraic eigenvalue problem. Its determinant must be zero for nontrivial solutions to exist, leading to a dispersion relation. This algebraic equation, however, is much more complex than before since the matrix formed is significantly less sparse. Hence, taking its derivatives with respect to the wavenumbers and applying the zero group velocity conditions to generate auxiliary dispersion relations is a much more computationally intensive task. As a consequence, grid convergence often becomes an issue that prevents two and three-dimensional absolute instabilities from being investigated, although low accuracy low dimensional approximations are commonly used.

(b) One two-dimensional branch point singularity is found and causality is proved, usually using the collision criterion. Then continuation techniques are employed to both search for additional singularities over the parametric space and extend this search towards three-dimensional singularities. This technique calculates temporal growth rates in a reference frame moving with the wave packet group velocities. If these velocities at the marginal stability conditions have the same sign, the flow is convectively unstable. Otherwise it is absolutely unstable. Such a procedure requires lab reference frame temporal growth rates and their respective group velocities within a large complex wavenumber set, making them expensive. Furthermore, it can only capture singularities connected by continuity. A significantly more efficient version of this procedure equates the numerically obtained group velocities to zero and uses these equations as auxiliary differential eigenvalue problems. Since these auxiliary problems are only satisfied at branch point singularities, root-finding procedures can be employed to automatically search for these points. 
In the vast majority of cases, however, this procedure has been applied only to two-dimensional problems.

For the reasons discussed above, a determination of the convective/absolute nature of a linear stability problem defined by one or more ordinary differential equations, i.e. differential eigenvalue problems, has been restricted to two or pseudo-two dimensional disturbances in most cases. On the other hand, a few recent studies have shown that three dimensional disturbances can behave quite differently than their two and pseudotwo dimensional counterparts and, hence, must be investigated as well. A solution to this problem was presented here with the theoretical development of a new method using sensitivity analysis through the following steps:

(i) The derivative of the differential eigenvalue problem with respect to each wavenumber generates auxiliary differential eigenvalue problems that model its sensitivity with respect to these wavenumbers.

(ii) Allowing the frequency derivatives with respect to the wavenumbers that appear in these auxiliary differential eigenvalue problems to be zero couples these differential eigenvalue problems at branch points.

(iii) Recognizing that each differential eigenvalue problem yields one complex eigenfunction allows one to define the eigenfunction derivatives with respect to the wavenumbers as unknown eigenfunctions themselves. Hence, the direct differential eigenvalue problem and (one) two auxiliary differential eigenvalue problems can be used to solve for (two) three eigenfunctions associated with (two) three-dimensional branch points.

(iv) Recognizing the fact that each differential eigenvalue problem also yields one complex eigenvalue allows the original differential eigenvalue problem and (one) two auxiliary differential eigenvalue problems to yield the coupled frequency and (one) two wavenumbers associated with (two) three-dimensional branch points.

(v) The auxiliary differential eigenvalue problems were proven to be the differential counterparts of the algebraic auxiliary dispersion relations.

(vi) Since the auxiliary differential eigenvalue problems are inhomogeneous boundary value problems, solvability conditions can be invoked to guarantee solution existence. They can be re-written as explicit integral formulas for the group velocities.

(vii) When the linear and homogeneous operator of the differential eigenvalue problem can be written in symmetrical form, the adjoint eigenfunction in the solvability condition can be replaced by the complex conjugate of the direct eigenfunction, eliminating the need to solve the adjoint problem when calculating the group velocities.

Numerical simulations of three different differential eigenvalue problems to search for two, pseudo two and three-dimensional branch points led to the following discoveries:

(i) When coupled with the auxiliary differential eigenvalue problems, the differential eigenvalue problem solution provides two, pseudo two and three-dimensional branch point singularities with user prescribed accuracy.

(ii) It is not yet clear how these coupled differential eigenvalue problems can be solved using matrix forming and eigenvalue solvers. Hence, shooting methods are required.

(iii) Excluding the original proof of causality, which requires deforming integration contours to pass through branch point singularities, collision checks are arguably the most inefficient way of searching for two and three-dimensional singularities.

(iv) Matrix forming the differential eigenvalue problem, forcing the resulting matrix determinant to be zero to generate a dispersion relation and taking its derivative with respect to the wavenumbers to generate the auxiliary dispersion relations is arguably the second most inefficient way of searching for branch point singularities when low/medium to high accuracy is desired. It is only efficient when very low/low accuracy is sufficient.

(v) The new method proposed here appears to be one order of magnitude faster than 
continuation methods based on numerical group velocity calculations, approximately, independent of the user prescribed error tolerance.

(vi) The new method proposed here appears to lose less efficiency than continuation methods based on numerical group velocity calculations when the search for branch point singularities is extended from two to three-dimensional problems.

(vii) The last two conclusions imply that enforcing the zero group velocity conditions through auxiliary differential eigenvalue problems, akin to what is done when using auxiliary dispersion relations, is more efficient than using these conditions themselves as the auxiliary differential eigenvalue problems.

(viii) The new method proposed here appears to be the most efficient procedure to search for two and three-dimensional branch point singularities whenever low/medium to high accuracy is desired.

Future work aims at further exploring the use of sensitivity analysis on differential eigenvalue problems in order to pursue both base flow and flow geometry optimization as well as the identification of wave makers. Furthermore, the authors are still trying to find a way to solve the coupled differential eigenvalue problems using matrix forming and eigenvalue solvers, as opposed to shooting methods. Doing so will greatly facilitate the identification of absolute instabilities in steady and local two-dimensional base flows.

\section{Acknowledgements}

Mateus Schuabb and Leo Alves would like to thank CNPq and CAPEs for the undergraduate and graduate fellowships that allowed the former to work on this project. Leo Alves, Silvia Hirata and Antonio Barletta would also like to thank CNPq, through grant 481072/2012-8, IME, Université Lille 1 and Università di Bologna for funding their respective visiting appointments over the past few years, which led to this work.

\section{REFERENCES}

Alves, L. S. De B., Kelly, R. E. \& Karagozian, A. R. 2008 Transverse jet shear layer instabilities. part ii: Linear analysis for large jet-to-crossflow velocity ratios. Journal of Fluid Mechanics 602, 383-401.

Alves, L. S.de B. \& Barletta, A. 2015 Convective to absolute instability transition in the Prats flow of a power-law fluid. International Journal of Thermal Sciences 94, 270-282.

Alves, L. S.de B., Hirata, S.da C. \& Ouarzazi, M. N. 2016 Linear onset of convective instability for Rayleigh-Bénard-Couette flows of viscoelastic fluids. Journal of NonNewtonian Fluid Mechanics 231, 79-90.

Benjamin, T. B. 1961 The development of three-dimensional disturbances in an unstable film of liquid flowing down an inclined plane. Journal of Fluid Mechanics 10, 401-419.

Bers, A. 1975 Physique des Plasmas, chap. Linear waves and instabilities, pp. 117-215. Gordon and Breach Science Publishers S.A.

Bers, A. 1983 Basic Plasma Physics, , vol. 1, chap. Space-time evolution of plasma instabilities - absolute and convective, pp. 451-517. Amsterdam: North-Holland Publishing Company.

Betchov, R. \& Criminale, W. O. 1966 Spatial instability of the inviscid jet and wake. Physics of Fluids 9 (2), 359-362.

Bottaro, A., Corbett, P. \& Luchini, P. 2003 The effect of base flow variation on flow stability. Journal of Fluid Mechanics 476, 293-302.

Brandao, P. V., Alves, L. S.de B. \& Barletta, A. 2014 Onset of absolute instability induced by viscous dissipation in the Poiseuille-Darcy-Bénard convection of a Newtonian fluid. Journal of Physics: Conference Series 547 (012039).

Brandao, P. V., Alves, L. S.de B. \& Barletta, A. 2015 Onset of mixed convection in a porous medium due to an absolute instability induced by viscous dissipation. In Bifurcations and Instabilities in Fluid Dynamics. ESPCI, Paris, France. 
Brevdo, L. 1991 Three-dimensional absolute and convective instabilities, and spatially amplifying waves in parallel shear flows. Journal of Applied Mathematics and Physics 42, 911-942.

Brevdo, L. 1995 Convectively unstable wave packets in the Blasius boundary layer. Zeitschrift für Angewandte Mathematik und Mechanik 75 (6), 423-436.

Brevdo, L. 2009 Three-dimensional absolute and convective instabilities at the onset of convection in a porous medium with inclined temperature gradient and vertical throughflow. Journal of Fluid Mechanics 641, 475-487.

Brevdo, L., Laure, P., Dias, F. \& Bridges, T. J. 1999 Linear pulse structure and signalling in a film flow on an inclined plane. Journal of Fluid Mechanics 396, 37-71.

Brevdo, L. \& Ruderman, M. S. 2009 On the convection in a porous medium with inclined temperature gradient and vertical throughflow. part ii. absolute and convective instabilities, and spatially amplifying waves. Transport in Porous Media 80, 153-172.

Bridges, T. J. \& Morris, P. J. 1984 Differential eigenvalue problems in which the parameter appears nonlinearly. Journal of Computational Physics 55, 437-460.

Briggs, R. J. 1964 Electron-stream interaction with plasmas. Research Monograph 29. MIT.

Carrière, P. \& Monkewitz, P. A. 1999 Convective versus absolute instability in mixed Rayleigh-Bénard-Poiseuille convection. Journal of Fluid Mechanics 384, 243-262.

Chomaz, J. M., Couniron, A. \& Julien, S. 1999 Absolute and convective nature of the Eckhaus and zigzag instability with throughflow. Physics of Fluids 11 (11), 3369-3373.

Criminale, W. O. \& Kovasznay, L. S. G. 1962 The growth of localized disturbances in a laminar boundary layer. Journal of Fluid Mechanics 14, 59-80.

Deissler, R. J. 1987 The convective nature of instability in plane Poiseuille flow. Physics of Fluids 30, 2303-2305.

Delache, A, Ouarzazi, M. N. \& Combarnous, M. 2007 Spatio-temporal stability analysis of mixed convection flows in porous media heated from below: Comparison with experiments. International Journal of Heat and Mass Transfer 50, 1485-1499.

Gaster, M. 1968 Growth of disturbances in both space and time. Physics of Fluids 11, 723-727.

Hirata, S.da C., Alves, L. S.de B., Delenda, N. \& Ouarzazi, M. N. 2015 Convective and absolute instabilities in Rayleigh-Bénard-Poiseuille mixed convection for viscoelastic fluids. Journal of Fluid Mechanics 765, 167-210.

Hirata, S. C. \& OuARzAZI, M. N. 2010 Three-dimensional absolute and convective instabilities in mixed convection of a viscoelastic fluid through a porous medium. Physics Letters, Section A: General, Atomic and Solid State Physics 374 (26), 2661-2666.

Huerre, P. \& Monkewitz, P. A. 1985 Absolute and convective instabilities in free shear layers. Journal of Fluid Mechanics 159, 151-168.

Huerre, P. \& Monkewitz, P. A. 1990 Local and global instabilities in spatially developing flows. Annual Review of Fluid Mechanics 22, 473-537.

JuniPeR, M. P. \& PieR, B. 2015 The structural sensitivity of open shear flows calculated with a local stability analysis. European Journal of Mechanics B/Fluids 49, 426-437.

Kelly, R. E. \& Alves, L. S. DE B. 2008 A uniformly valid asymptotic solution for the transverse jet and its linear stability analysis. Philosophical Transactions of the Royal Society of London. Series A: Mathematical and Physical Sciences 366, 2729-2744.

Koch, W. 2002 On the spatio-temporal stability of primary and secondary crossflow vortices in a three-dimensional boundary layer. Journal of Fluid Mechanics 456, 85-111.

Leclerce, C., Pier, B. \& Scott, J. F. 2014 Absolute instabilities in eccentric Taylor-CouettePoiseuille flow. Journal of Fluid Mechanics 741, 543-566.

Lesshafft, L. \& Marquet, O. 2010 Optimal velocity and density profiles for the onset of absolute instability in jets. Journal of Fluid Mechanics 662, 398-408.

Lingwood, R. J. 1995 Absolute instability of the boundary layer on a rotating disk. Journal of Fluid Mechanics 299, 17-33.

Lingwood, R. J. 1997 a Absolute instability of the Ekman layer and related rotating flows. Journal of Fluid Mechanics 331, 405-428.

LingwoOD, R. J. $1997 b$ On the application of the Briggs' and steepest-descent methods to a boundary-layer flow. Studies in Applied Mathematics 98 (3), 213-254.

LingwOOD, R. J. 1997c On the impulse response for swept boundary-layer flows. Journal of Fluid Mechanics 344, 317-334. 
Luchini, P. \& Bottaro, A. 2014 Adjoint equations in stability analysis. Annual Review of Fluid Mechanics 46, 493-517.

Martinand, D., Carrière, P. \& Monkewitz, P. A. 2004 Envelope equations for the Rayleigh-Bénard-Poiseuille system. part 2. linear global modes in the case of twodimensional non-uniform heating. Journal of Fluid Mechanics 502, 175-197.

Merkine, L. O. 1977 Convective and absolute instability of baroclinic eddies. Geophysical \& Astrophysical Fluid Dynamics 9, 129-157.

Monkewitz, P. A. \& Nguyen, L. N. 1987 Absolute instability in the near-wake of two dimensional bluff bodies. Journal of Fluids and Structures 1 (165-184).

Monkewitz, P. A. \& Sohn, K. D. 1988 Absolute instability in hot jets. AIAA Journal 26 (8), 911-916.

Oertel JR., H. \& Delfs, J. 1995 Mathematische analyse der bereiche reibungsbehafteter strömungen. Zeitschrift für Angewandte Mathematik und Mechanik 75, 491-505.

Pier, B. 2009 Open-loop control of absolutely unstable domains. Proceedings of the Royal Society of London. Series A, Mathematical and Physical Sciences 459, 1105-1115.

Schlichting, H. 1986 Boundary-Layer Theory, 7th edn. New York: MacGraw Hill, Inc.

Strang, G. 1988 Linear Algebra and its Applications, 3rd edn. Harcourt Brace Jovanovich.

Suslov, S. A. 2006 Numerical aspects of searching convective/absolute instability transition. Journal of Computational Physics 212, 188-217.

Thacker, W. C. 1976 Spatial growth of gulf stream meanders. Geophysical Fluid Dynamics 7, 271-295.

Theofilis, V. 2003 Advances in global linear instability analysis of nonparallel and threedimensional flows. Progress in Aerospace Sciences 39, 249-315.

Theofilis, V. 2011 Global linear instability. Annual Review of Fluid Mechanics 43, 319-352.

Wolfram, S. 2003 The Mathematica Book, 5th edn. New York: Cambridge University Press. 